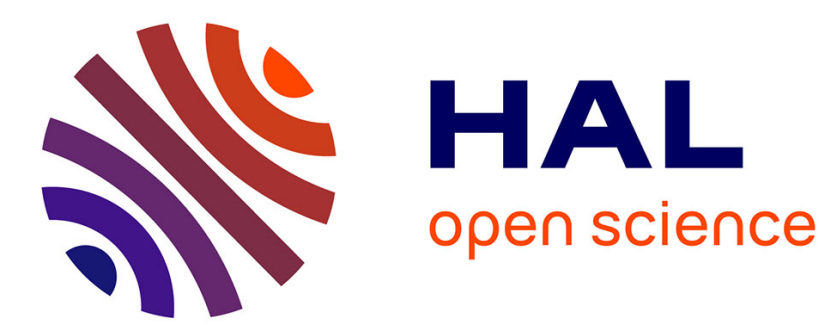

\title{
On the influence of wavy riblets on the slip behaviour of viscous fluids
}

\author{
Matthieu Bonnivard, Francisco J Suárez-Grau, Giordano Tierra
}

\section{To cite this version:}

Matthieu Bonnivard, Francisco J Suárez-Grau, Giordano Tierra. On the influence of wavy riblets on the slip behaviour of viscous fluids. Zeitschrift für Angewandte Mathematik und Physik, 2016, 67, pp.27. 10.1007/s00033-015-0614-y . hal-01471769

\section{HAL Id: hal-01471769 \\ https://hal.sorbonne-universite.fr/hal-01471769}

Submitted on 20 Feb 2017

HAL is a multi-disciplinary open access archive for the deposit and dissemination of scientific research documents, whether they are published or not. The documents may come from teaching and research institutions in France or abroad, or from public or private research centers.
L'archive ouverte pluridisciplinaire $\mathbf{H A L}$, est destinée au dépôt et à la diffusion de documents scientifiques de niveau recherche, publiés ou non, émanant des établissements d'enseignement et de recherche français ou étrangers, des laboratoires publics ou privés. 


\title{
ON THE INFLUENCE OF WAVY RIBLETS ON THE SLIP BEHAVIOUR OF VISCOUS FLUIDS
}

\author{
Matthieu Bonnivard*, Francisco J. Suárez-Grau * ${ }^{*}$ \& Giordano Tierra ${ }^{\ddagger}$
}

September 14, 2016

\begin{abstract}
In this work, we use the homogenization theory to investigate the capability of wavy riblet patterns to influence the behaviour of a viscous flow near a ribbed boundary. Starting from perfect slip conditions on the wall, we show that periodic oscillations of wavy riblets in the lateral direction may induce a friction effect in the direction of the flow, contrary to what happens with straight riblets. Finally, we illustrate this effect numerically by simulating riblet profiles that are widely used in experimental studies: the $V$-shape, $U$-shape, and blade riblets.
\end{abstract}

Keywords: viscous fluids, slip condition, rough boundary, straight riblets, wavy riblets, homogenization.

Mathematics subject classifications (2000): 35B27, 35Q35, 74A55.

\section{Contents}

1 Introduction 2

2 Notation 5

$\begin{array}{lll}3 & \text { Setting of the problem and main result } & 7\end{array}$

4 Proofs of the results $\quad 10$

5 Numerical results $\quad 21$

5.1 Numerical approximation of auxiliary system $(3.14) \ldots \ldots$. . . . . . 21

5.2 Definition of the geometry of the wavy riblets . . . . . . . . . . 22

5.3 Results and discussion . . . . . . . . . . . . . . . . 23

${ }^{*}$ Univ. Paris Diderot, Sorbonne Paris Cité, Laboratoire Jacques-Louis Lions, UMR 7598, UPMC, CNRS, F-75205 Paris France, email: bonnivard@ljll.univ-paris-diderot.fr

${ }^{\dagger}$ Dpto. Ecuaciones Diferenciales y Análisis Numérico, Universidad de Sevilla, Seville, 41012, Spain, e-mail: fjsgrau@us.es

${ }^{\ddagger}$ Mathematical Institute, Faculty of Mathematics and Physics, Charles University, Prague 8, 18675 , Czech Republic, e-mail: gtierra@karlin.mff.cuni.cz 


\section{Introduction}

During the last decades, the reduction of the skin friction associated with a viscous flow in the vicinity of a solid boundary has been the subject to an abundant literature. From an experimental point of view, an efficient method to reduce the friction consists in using a certain type of rough boundaries, that are called riblets or straight riblets. These riblets are characterized by fast oscillations in the spanwise direction, with a low amplitude, and by their constancy in the streamwise direction; they are essentially onedimensional perturbations of the boundary of the solid. The spanwise geometry of the riblets is generally described by the periodic repetition of a concave pattern, typically, the shape of a $\mathrm{V}$ or a $\mathrm{U}$. The aim of the use of riblets is to prevent vortices to appear in the neighborhood of the solid wall, and thus to reduce the momentum transfer from the vortices to the solid boundary.

Recently, new methods have been investigated to enhance the efficiency of the riblets. One of these methods consists in a combination of riblets with an oscillating wall, that is achieved by tilting riblet blades sinusoidally. This approach has improved the drag reduction from $8.6 \%$ to $11.1 \%$ [30]. However, this result needs to be put in perspective, since the temporal oscillations of the boundary require a certain input of energy into the system.

Another possible approach consists in using spatial oscillations of the riblets in the streamwise direction, thus replacing a temporal, active process by a static, passive one. This method was studied experimentally in [29], with a net power reduction increased up to $23 \%$. The corresponding devices are called wavy riblets; they consist in straight riblets that are deflected sinusoidally in the spanwise direction, as a function of the streamwise position. Wavy riblets were studied in [25], both numerically and experimentally, in the case of a turbulent flow; however, the authors were not able to conclude regarding the efficiency of the device.

In the present paper, we study the effect of wavy riblets on the slip behaviour of a viscous flow, when the amplitude and the periods of the spanwise and streamwise oscillations are small. Although the main field of application of wavy riblets concerns high Reynolds number flows, the present work deals with the case of low Reynolds number, in which the flow can be modeled via the linear Stokes equations. We believe that the presented results could be instrumental in the mathematical study of certain models for turbulence used in Large Eddy Simulation, that we aim to address in a future work.

Definition of straight and wavy riblets. The ribbed walls that we consider are geometric perturbations of a planar boundary, which is identified with the plane $\mathbb{R}^{2} \times\{0\}$. In the cartesian system of coordinates $\left(x_{1}, x_{2}, x_{3}\right), x_{3}$ denotes the wall-normal coordinate. We define $x_{2}$ as the direction of the flow, or streamwise direction; $x_{1}$ is thus the lateral, or spanwise direction.

We assume that the geometry of the riblets is associated with a small parameter $\varepsilon$, that controls the (spatial) oscillations of the boundary. More precisely, we name wavy riblets a parametric surface defined by a relation of the form $x_{3}=-\Psi_{\varepsilon}\left(x_{1}, x_{2}\right)$, where $\Psi_{\varepsilon}$ is a positive function given by

$$
\Psi_{\varepsilon}\left(x_{1}, x_{2}\right):=a_{\varepsilon}^{1} \Psi_{1}\left(\frac{x_{1}}{p_{\varepsilon}^{1}}+\frac{a_{\varepsilon}^{2}}{p_{\varepsilon}^{1}} \Psi_{2}\left(\frac{x_{2}}{p_{\varepsilon}^{2}}\right)\right),
$$

$\Psi_{1}, \Psi_{2}$ being smooth and periodic functions of period 1. Parameters $a_{\varepsilon}^{1}, p_{\varepsilon}^{1}>0$ (resp. 
$a_{\varepsilon}^{2}, p_{\varepsilon}^{2}>0$ ) are the amplitude and period of oscillation in the $x_{1}-x_{3}$ axes (resp., in the $x_{1}-x_{2}$ axes). Let us stress that the definition of wavy riblets (1.1) is a generalization of straight riblets, which correspond to the choice $\Psi_{2} \equiv 0$ (see Figure 1).
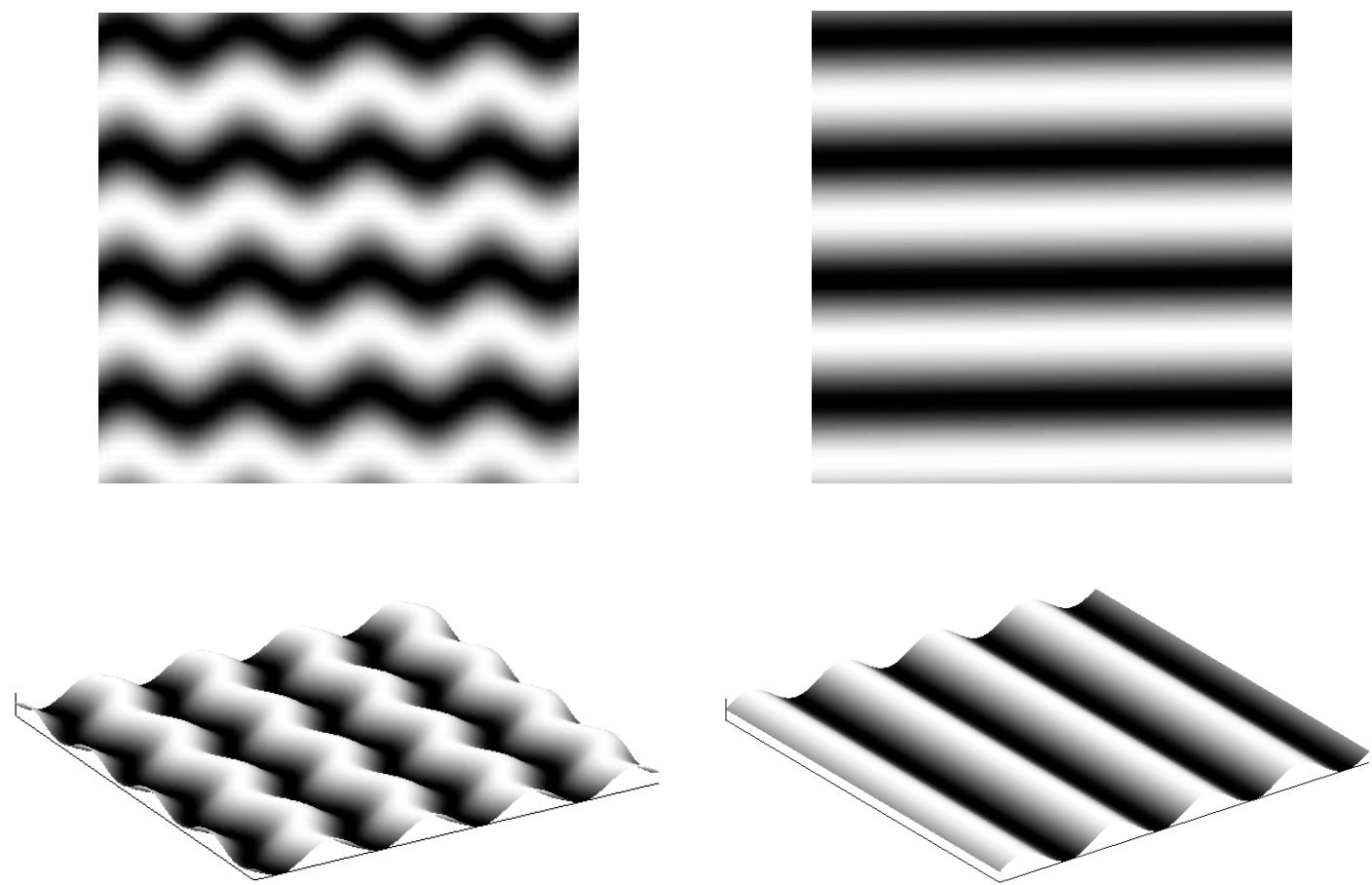

Figure 1: Views from above of wavy riblets (left) and straight riblets (right)

In order to study the rugosity effect of straight riblets endowed with perfect slip conditions, several theoretical works related to homogenization theory have been carried out. It was shown in [7] that, starting with perfect slip condition at the ribbed boundary given by

$$
\Psi_{\varepsilon}\left(x_{1}, x_{2}\right)=\varepsilon \Psi\left(\frac{x_{1}}{\varepsilon}\right),
$$

in the limit $\varepsilon \rightarrow 0$, no-slip condition appears in the spanwise direction while perfect slip still holds in the streamwise direction. This means that straight riblets tend to prevent the fluid from slipping laterally, whereas the motion in the streamwise direction is allowed with no constraint. The former work was generalized in [15] for riblets with low amplitude, i.e.

$$
\Psi_{\varepsilon}\left(x_{1}, x_{2}\right)=\delta_{\varepsilon} \Psi\left(\frac{x_{1}}{\varepsilon}\right),
$$

where $\delta_{\varepsilon} \ll \varepsilon$. In that case, the limit velocity field satisfies a perfect slip condition in the streamwise direction, but its slippage behaviour in the spanwise direction is dependant on the scaling of $\delta_{\varepsilon}$ with respect to the critical scaling $\varepsilon^{\frac{3}{2}}$ :

- if $\delta_{\varepsilon} \ll \varepsilon^{\frac{3}{2}}$, then there is no roughness effect and perfect slip is achieved in the spanwise direction as well;

- if $\delta_{\varepsilon} \approx \varepsilon^{\frac{3}{2}}$, with $\delta_{\varepsilon} / \varepsilon^{\frac{3}{2}} \rightarrow \lambda, 0<\lambda<+\infty$, then partial slip appears in the spanwise direction, which is associated with a constant friction coefficient depending on the 
limit $\lambda$ and on the riblet geometry;

- if $\delta_{\varepsilon} \gg \varepsilon^{\frac{3}{2}}$, then the same consequences given in [7] hold: no-slip condition appears in the spanwise direction while perfect slip holds in the streamwise one.

The common feature of these previous works is that the rugosity effect induced by straight riblets can only appear in the spanwise direction. Thus, our goal in the present paper is to analyse if the periodic displacement of wavy riblets in the lateral direction may produce an effect on the streamwise component of the velocity. For the sake of simplicity, in this work we will consider the same amplitudes and periods in both the streamwise and spanwise directions, by setting $a_{\varepsilon}^{1}=a_{\varepsilon}^{2}=\delta_{\varepsilon}, p_{\varepsilon}^{1}=p_{\varepsilon}^{2}=\varepsilon$, where $\delta_{\varepsilon} \ll \varepsilon$ as $\varepsilon$ tends to 0 . The function $\Psi_{\varepsilon}$ will thus be defined by (see Figure 2)

$$
\Psi_{\varepsilon}\left(x_{1}, x_{2}\right)=\delta_{\varepsilon} \Psi_{1}\left(\frac{x_{1}}{\varepsilon}+\frac{\delta_{\varepsilon}}{\varepsilon} \Psi_{2}\left(\frac{x_{2}}{\varepsilon}\right)\right) .
$$
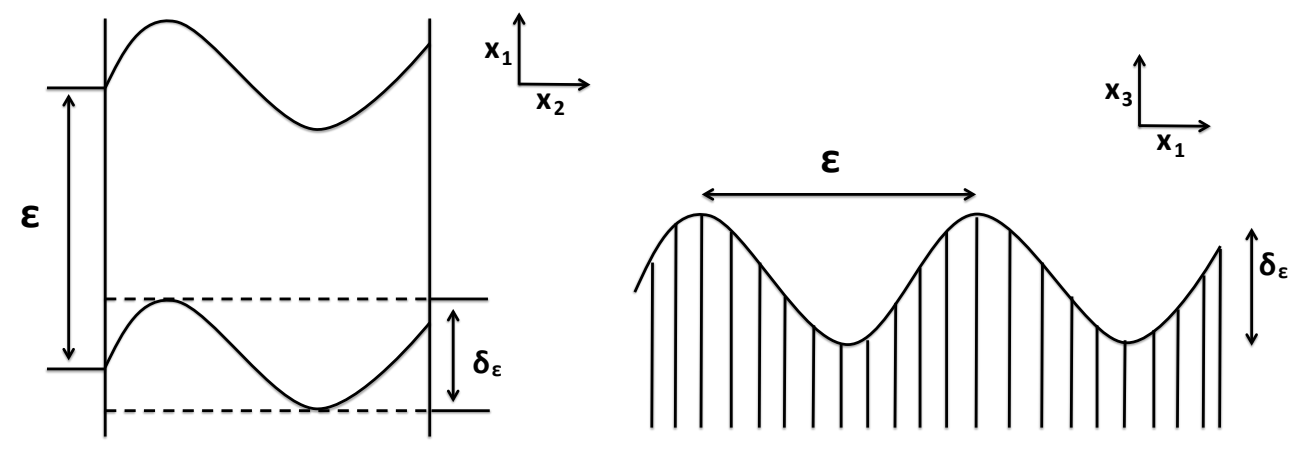

Figure 2: Sketch of sinusoidal displacement. View of wavy riblet surface from above (left) and wavy riblet cross-section (right)

For such configuration, we prove that the asymptotic effects of wavy riblets also depend on the scaling of $\delta_{\varepsilon}$ with respect to $\varepsilon$; however, two critical regimes need to be considered, namely $\delta_{\varepsilon} \approx \varepsilon^{\frac{3}{2}}$ and $\delta_{\varepsilon} \approx \varepsilon^{\frac{5}{4}}$, and therefore five different regimes. As it was the case in [15], the critical regime $\delta_{\varepsilon} \approx \varepsilon^{\frac{3}{2}}$ is associated with the effect of the riblets in the spanwise direction. The novelty of the model we consider is that the combination of streamwise and spanwise oscillations of wavy riblets gives rise to a new critical regime $\delta_{\varepsilon} \approx \varepsilon^{\frac{5}{4}}$, that rules the asymptotic effect of the geometry on the streamwise component of the fluid velocity. Namely, the conclusion given in [7] for $\delta_{\varepsilon} \gg \varepsilon^{\frac{3}{2}}$ is replaced by two additional asymptotic regimes:

- if $\delta_{\varepsilon} \approx \varepsilon^{\frac{5}{4}}$, with $\delta_{\varepsilon} / \varepsilon^{\frac{5}{4}} \rightarrow \mu, 0<\mu<+\infty$, then no-slip holds in the spanwise direction, while partial slip described by a constant friction coefficient $M_{\mu}$ appears in the streamwise direction;

- if $\delta_{\varepsilon} \gg \varepsilon^{\frac{5}{4}}$, then the periodical displacement of wavy riblets in the spanwise direction enforces the fluid to satisfy no-slip condition in both directions. 
Let us stress that the case $\delta_{\varepsilon} \gg \varepsilon^{\frac{5}{4}}$, where perfect slip condition transforms asymptotically in no-slip condition, is an example of the strongest rugosity effect: the roughness prevents the fluid from slipping on the wall. This effect was identified in the pioneering paper [13], in the case of a periodic, self-similar boundary with $C^{2}$-regularity. This regularity was weakened to "almost Lipschitz" boundaries in [6]. Other results obtained in [9], [10] (see also [23], [26]) give a quite complete understanding of the rugosity effect for arbitrary boundaries.

The paper is organized as follows. In Section 2, we introduce the notation. In Section 3, we formulate the problem and state our main result, which is proved in Section 4 by means of an adaptation of the unfolding method (see e.g. [3], [11], [14], [16]), that was proposed in [15]. Finally, in Section 5, we illustrate numerically the asymptotic effect of wavy riblets on the streamwise component of the velocity field. To this aim, we simulate the friction coefficient $M_{\mu}$ induced by the critical regime $\delta_{\varepsilon} \approx \varepsilon^{\frac{5}{4}}$, and the corresponding homogenized system, using different riblet profiles that are widely used in the experimental studies: the $V$-shape, the $U$-shape, and the blade riblets.

\section{Notation}

We consider a viscous flow in a domain delimited by two horizontal plates. For the sake of simplicity, we assume that the flow is periodic with respect to the horizontal coordinates $\left(x_{1}, x_{2}\right)$. We denote by $\mathcal{T}^{2}:=\mathbb{R}^{2} / \mathbb{Z}^{2}$ (resp. $\mathcal{T}^{1}:=\mathbb{R} / \mathbb{Z}$ ) the two-dimensional (resp. one-dimensional) torus. We fix two functions $\Psi_{1}, \Psi_{2} \in W^{2, \infty}\left(\mathcal{T}^{1}\right)$ and define

$$
\Psi_{\varepsilon}\left(x_{1}, x_{2}\right):=\delta_{\varepsilon} \Psi_{1}\left(\frac{x_{1}}{\varepsilon}+\frac{\delta_{\varepsilon}}{\varepsilon} \Psi_{2}\left(\frac{x_{2}}{\varepsilon}\right)\right), \quad \text { a.e. }\left(x_{1}, x_{2}\right) \in \mathcal{T}^{2},
$$

where $\varepsilon>0$ is a small parameter and $\delta_{\varepsilon}$ satisfies

$$
\lim _{\varepsilon \rightarrow 0} \delta_{\varepsilon}=0, \quad \lim _{\varepsilon \rightarrow 0} \frac{\delta_{\varepsilon}}{\varepsilon}=0 .
$$

We consider the spatial domain

$$
\Omega_{\varepsilon}:=\left\{\left(x_{1}, x_{2}, x_{3}\right) \in \mathcal{T}^{2} \times \mathbb{R}, \quad-\Psi_{\varepsilon}\left(x_{1}, x_{2}\right)<x_{3}<1\right\} .
$$

The two components of the boundary $\partial \Omega_{\varepsilon}$ are denoted by

$$
\begin{gathered}
\Gamma_{\text {top }}:=\mathcal{T}^{2} \times\{1\}, \\
\Gamma_{\varepsilon}:=\left\{\left(x_{1}, x_{2}, x_{3}\right) \in \mathcal{T}^{2} \times \mathbb{R}, \quad x_{3}=-\Psi_{\varepsilon}\left(x_{1}, x_{2}\right)\right\} .
\end{gathered}
$$

With these definitions, the upper surface $\Gamma_{\text {top }}$ is assumed to be plane, while the lower one, $\Gamma_{\varepsilon}$, is composed of wavy riblets (see Figure 3). We also define the limit domain $\Omega$ and its plane bottom surface $\Gamma$ by

$$
\Omega:=\mathcal{T}^{2} \times(0,1), \quad \Gamma:=\mathcal{T}^{2} \times\{0\} .
$$

For $k^{\prime} \in \mathbb{Z}^{2}$, we denote

$$
C_{\varepsilon}^{k^{\prime}}=\varepsilon k^{\prime}+\varepsilon \mathcal{T}^{2}, \quad Q_{\varepsilon}^{k^{\prime}}=\Omega_{\varepsilon} \cap\left(C_{\varepsilon}^{k^{\prime}} \times(-\infty, 1)\right) .
$$




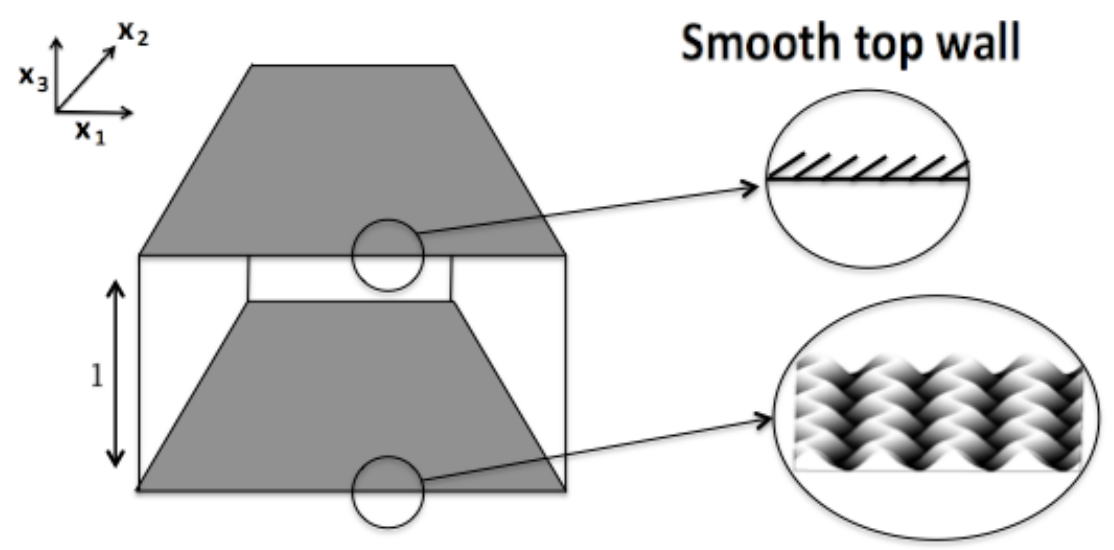

Bottom wall with wavy riblets

Figure 3: Schematic view of domain $\Omega_{\varepsilon}$

We define $\kappa: \mathcal{T}^{2} \rightarrow \mathbb{Z}^{2}$ by

$$
\kappa\left(x^{\prime}\right)=k^{\prime} \Leftrightarrow x^{\prime} \in C_{1}^{k^{\prime}}
$$

Remark that $\kappa$ is well defined up to a set of zero measure in $\mathcal{T}^{2}$ (the set $\cup_{k^{\prime} \in \mathbb{Z}^{2}} \partial C_{1}^{k^{\prime}}$ ). Moreover, for every $\varepsilon>0$, we have

$$
\kappa\left(\frac{x^{\prime}}{\varepsilon}\right)=k^{\prime} \Leftrightarrow x^{\prime} \in C_{\varepsilon}^{k^{\prime}} .
$$

For a.e. $x^{\prime} \in \mathcal{T}^{2}$ we define $C_{\varepsilon}\left(x^{\prime}\right)$ as the square $C_{\varepsilon}^{k^{\prime}}$ such that $x^{\prime}$ belongs to $C_{\varepsilon}^{k^{\prime}}$. For every $\varepsilon>0$, we define $I_{\varepsilon}$ by

$$
I_{\varepsilon}=\left\{k^{\prime} \in \mathbb{Z}^{2}: C_{\varepsilon}^{k^{\prime}} \cap \mathcal{T}^{2} \neq \emptyset\right\}
$$

We define the set $\widehat{Q}:=\mathcal{T}^{2} \times(0,+\infty)$, and for every $M>0$, we write $\widehat{Q}_{M}=\mathcal{T}^{2} \times(0, M)$ and we decompose each element $x \in \widehat{Q}_{M}$ in $x=\left(x^{\prime}, x_{3}\right)$, with obvious notation. We denote by $\mathcal{V}$ the space of functions $v: \widehat{Q} \rightarrow \mathbb{R}$ such that

$$
v \in H^{1}\left(\widehat{Q}_{M}\right) \quad \forall M>0, \quad \nabla v \in L^{2}(\widehat{Q})^{3} .
$$

$\mathcal{V}$ is a Hilbert space endowed with the norm $\|\cdot\|_{\mathcal{V}}$ defined by

$$
\|v\|_{\mathcal{V}}^{2}=\|v\|_{L^{2}\left(\mathcal{T}^{2} \times\{0\}\right)}^{2}+\|\nabla v\|_{L^{2}(\widehat{Q})}^{2} .
$$

For a bounded measurable set $\Theta \subset \mathbb{R}^{N}$, we denote by $L_{0}^{2}(\Theta)$ the space of functions of $L^{2}(\Theta)$ with null integral.

We denote by $O_{\varepsilon}$ a generic real sequence which tends to zero with $\varepsilon$ and can change from line to line. We denote by $C$ a generic positive constant which can change from line to line. 


\section{Setting of the problem and main result}

Let $\Omega_{\varepsilon}$ be the domain defined by (2.2). We consider the Stokes equations

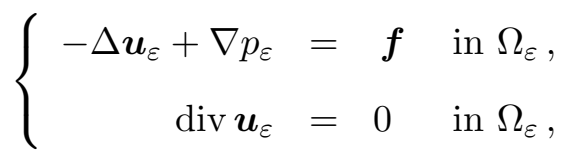

where the unknown functions $\boldsymbol{u}_{\varepsilon}$ and $p_{\varepsilon}$ are the velocity and the pressure of the fluid, respectively. Note that we have tacitly assumed that the driving force $\boldsymbol{f}$ is defined on all domains $\Omega_{\varepsilon}$, say, $\boldsymbol{f}$ is a restriction of a fixed function belonging to the class $L^{\infty}\left(\mathcal{T}^{2} \times \mathbb{R}\right)^{3}$. We assume for simplicity that the fluid adheres to the top boundary, that means,

$$
\boldsymbol{u}_{\varepsilon}=\mathbf{0} \quad \text { on } \Gamma_{\text {top }} .
$$

The aim is to study the roughness-induced effects of wavy riblets at the bottom of the domain. In view of that, we assume impermeability of the lower surface

$$
\boldsymbol{u}_{\varepsilon} \cdot \boldsymbol{\nu}=0 \text { on } \Gamma_{\varepsilon},
$$

together with the perfect slip condition

$$
\left[\frac{\partial \boldsymbol{u}_{\varepsilon}}{\partial \boldsymbol{\nu}}\right]_{\boldsymbol{\tau}}=0 \text { on } \Gamma_{\varepsilon}
$$

where $\boldsymbol{\nu}$ denotes the outside unitary normal vector to $\Omega_{\varepsilon}$ on $\Gamma_{\varepsilon}$, and []$_{\boldsymbol{\tau}}$ denotes the tangential component.

It is well known (see e.g. [7], [15]) that problem (3.1) has a unique solution $\left(\boldsymbol{u}_{\varepsilon}, p_{\varepsilon}\right) \in$ $H^{1}\left(\Omega_{\varepsilon}\right)^{3} \times L_{0}^{2}\left(\Omega_{\varepsilon}\right)$, and moreover there exists $C>0$, which does not depend on $\varepsilon$, such that

$$
\left\|\boldsymbol{u}_{\varepsilon}\right\|_{H^{1}\left(\Omega_{\varepsilon}\right)^{3}}+\left\|p_{\varepsilon}\right\|_{L^{2}\left(\Omega_{\varepsilon}\right)} \leq C .
$$

We are now in a position to state the main result of this paper, which describes the asymptotic behaviour of the solution $\left(\boldsymbol{u}_{\varepsilon}, p_{\varepsilon}\right)$ of problem (3.1)-(3.4). The problem satisfied by the limit $(\boldsymbol{u}, p)$ still possesses the same structure (see formula (3.7)), because the roughness-induced effect appears only on the boundary condition on the limit lower surface $\Gamma$. This effect depends on the asymptotic behaviour of $\delta_{\varepsilon}$, with two critical regimes, namely $\delta_{\varepsilon} \approx \varepsilon^{\frac{3}{2}}$, and $\delta_{\varepsilon} \approx \varepsilon^{\frac{5}{4}}$, and therefore five different regimes.

Theorem 3.1. Let $\left(\boldsymbol{u}_{\varepsilon}, p_{\varepsilon}\right) \in H^{1}\left(\Omega_{\varepsilon}\right)^{3} \times L_{0}^{2}\left(\Omega_{\varepsilon}\right)$ be the solution to problem (3.1) such that (3.5) holds. Then, there exists $(\boldsymbol{u}, p) \in H^{1}(\Omega)^{3} \times L_{0}^{2}(\Omega)$ such that, up to a subsequence,

$$
\boldsymbol{u}_{\varepsilon} \rightarrow \boldsymbol{u} \text { in } H^{1}(\Omega)^{3}, \quad p_{\varepsilon} \rightarrow p \text { in } L^{2}(\Omega) .
$$

The pair $(\boldsymbol{u}, p)$ satisfies the Stokes system

$$
\left\{\begin{array}{rlll}
-\Delta \boldsymbol{u}+\nabla p & = & \boldsymbol{f} & \text { in } \Omega, \\
\operatorname{div} \boldsymbol{u} & =0 & & \text { in } \Omega,
\end{array}\right.
$$

together with the boundary condition

$$
\boldsymbol{u}=\mathbf{0} \text { on } \Gamma_{\text {top }} .
$$

Moreover, it also satisfies the following boundary condition on $\Gamma$ depending on the asymptotic behaviour of $\delta_{\varepsilon}$ : 
(i) If $\delta_{\varepsilon} \ll \varepsilon^{\frac{3}{2}}$, then

$$
-\partial_{3} u_{1}=0, \quad-\partial_{3} u_{2}=0, \quad u_{3}=0, \quad \text { on } \Gamma .
$$

(ii) If $\delta_{\varepsilon} \approx \varepsilon^{\frac{3}{2}}$, with $\delta_{\varepsilon} / \varepsilon^{\frac{3}{2}} \rightarrow \lambda, 0<\lambda<+\infty$, we define $\left(\widehat{\phi}^{\lambda}, \widehat{q}^{\lambda}\right) \in \mathcal{V}^{3} \times L^{2}(\widehat{Q})$, as the solution of

$$
\left\{\begin{array}{rrr}
-\Delta_{y} \widehat{\boldsymbol{\phi}}^{\lambda}+\nabla_{y} \widehat{q}^{\lambda}=\mathbf{0} & \text { in } & \mathbb{R}^{2} \times \mathbb{R}^{+}, \\
\operatorname{div}_{y} \widehat{\boldsymbol{\phi}}^{\lambda}=0 & \text { in } & \mathbb{R}^{2} \times \mathbb{R}^{+}, \\
\widehat{\phi}_{3}^{\lambda}\left(y^{\prime}, 0\right)=\lambda \partial_{1} \Psi_{1}\left(y_{1}\right) & \text { on } & \mathbb{R}^{2} \times\{0\}, \\
-\partial_{3} \widehat{\phi}_{1}^{\lambda}=0,-\partial_{3} \widehat{\phi}_{2}^{\lambda}=0 & \text { on } & \mathbb{R}^{2} \times\{0\},
\end{array}\right.
$$

and $M_{\lambda} \in \mathbb{R}$ by

$$
M_{\lambda}=\int_{\widehat{Q}}\left|D_{y} \widehat{\phi}^{\lambda}\right|^{2} d y
$$

Then

$$
-\partial_{3} u_{1}+M_{\lambda} u_{1}=0, \quad-\partial_{3} u_{2}=0, \quad u_{3}=0 \quad \text { on } \Gamma \text {. }
$$

(iii) If $\varepsilon^{\frac{3}{2}} \ll \delta_{\varepsilon} \ll \varepsilon^{\frac{5}{4}}$, then

$$
u_{1}=0, \quad-\partial_{3} u_{2}=0, \quad u_{3}=0, \quad \text { on } \Gamma \text {. }
$$

(iv) If $\delta_{\varepsilon} \approx \varepsilon^{\frac{5}{4}}$, with $\delta_{\varepsilon} / \varepsilon^{\frac{5}{4}} \rightarrow \mu, 0<\mu<+\infty$, we define $\left(\widehat{\phi}^{\mu}, \widehat{q}^{\mu}\right) \in \mathcal{V}^{3} \times L^{2}(\widehat{Q})$, as the solution of

$$
\left\{\begin{array}{rrr}
-\Delta_{y} \widehat{\phi}^{\mu}+\nabla_{y} \widehat{q}^{\mu}=\mathbf{0} & \text { in } & \mathbb{R}^{2} \times \mathbb{R}^{+}, \\
\operatorname{div}_{y} \widehat{\phi}^{\mu}=0 & \text { in } & \mathbb{R}^{2} \times \mathbb{R}^{+}, \\
\widehat{\phi}_{3}^{\mu}\left(y^{\prime}, 0\right)=\mu^{2} \partial_{1} \Psi_{1}\left(y_{1}\right) \partial_{2} \Psi_{2}\left(y_{2}\right) & \text { on } & \mathbb{R}^{2} \times\{0\}, \\
-\partial_{3} \widehat{\phi}_{1}^{\mu}=0,-\partial_{3} \widehat{\phi}_{2}^{\mu}=0, & \text { on } & \mathbb{R}^{2} \times\{0\},
\end{array}\right.
$$

and $M_{\mu} \in \mathbb{R}$ by

$$
M_{\mu}=\int_{\widehat{Q}}\left|D_{y} \widehat{\boldsymbol{\phi}}^{\mu}\right|^{2} d y
$$

Then

$$
u_{1}=0, \quad-\partial_{3} u_{2}+M_{\mu} u_{2}=0, \quad u_{3}=0, \quad \text { on } \Gamma \text {. }
$$

(v) If $\delta_{\varepsilon} \gg \varepsilon^{\frac{5}{4}}$, then

$$
u_{1}=0, \quad u_{2}=0, \quad u_{3}=0, \quad \text { on } \Gamma \text {. }
$$

Remark 3.2. Observe that in the first three cases, i.e. when $\delta_{\varepsilon} \ll \varepsilon^{\frac{5}{4}}$, roughness effects alter only the spanwise component of the velocity, $u_{1}$, while total slip is satisfied by the streamwise component, $u_{2}$. However, if $\delta_{\varepsilon} \gtrsim \varepsilon^{\frac{5}{4}}$, the wavy riblets have an influence on both components of the velocity; in the spanwise direction, the no-slip condition $u_{1}=0$ is enforced, and 
- If $\delta_{\varepsilon} \approx \varepsilon^{\frac{5}{4}}$, partial slip appears in the streamwise direction, associated with a constant friction coefficient $M_{\mu}$;

- If $\delta_{\varepsilon} \gg \varepsilon^{\frac{5}{4}}$, the no-slip condition $u_{2}=0$ is imposed asymptotically. In this case, the limit velocity field is completely adherent to the wall $\Gamma$.

Remark 3.3. Theorem 3.1 gives an approximation of $\left(\boldsymbol{u}_{\varepsilon}, p_{\varepsilon}\right)$ in the weak topology of $H^{1}(\Omega)^{3} \times L^{2}(\Omega)$. Following the proof of Theorem 3.3 in [15], we are able to obtain an approximation in the strong topologies (i.e., a corrector result). More precisely, in the non critical regimes, we can show that

$$
\int_{\Omega_{\varepsilon}}\left|\boldsymbol{u}_{\varepsilon}-\boldsymbol{u}\right|^{2} d x \rightarrow 0, \quad \int_{\Omega_{\varepsilon}}\left|D \boldsymbol{u}_{\varepsilon}-D \boldsymbol{u}\right|^{2} d x \rightarrow 0, \quad \int_{\Omega_{\varepsilon}}\left|p_{\varepsilon}-p\right|^{2} d x \rightarrow 0 .
$$

In the critical case $\delta_{\varepsilon} \approx \varepsilon^{\frac{3}{2}}$, defining $\boldsymbol{u}_{\varepsilon}^{\lambda}$ and $p_{\varepsilon}^{\lambda}$ by

$$
\boldsymbol{u}_{\varepsilon}^{\lambda}=\boldsymbol{u}(x)-\sqrt{\varepsilon} u_{1}\left(x^{\prime}, 0\right) \widehat{\phi}^{\lambda}\left(\frac{x}{\varepsilon}\right), \quad p_{\varepsilon}^{\lambda}=p(x)-\frac{1}{\sqrt{\varepsilon}} u_{1}\left(x^{\prime}, 0\right) \widehat{q}^{\lambda}\left(\frac{x}{\varepsilon}\right)
$$

then (3.18) still holds by replacing $\boldsymbol{u}$ and $p$ by $\boldsymbol{u}_{\varepsilon}^{\lambda}$ and $p_{\varepsilon}^{\lambda}$, respectively.

Finally, in the critical case $\delta_{\varepsilon} \approx \varepsilon^{\frac{5}{4}}$, defining $\boldsymbol{u}_{\varepsilon}^{\mu}$ and $p_{\varepsilon}^{\mu}$ by

$$
\boldsymbol{u}_{\varepsilon}^{\mu}=\boldsymbol{u}(x)-\sqrt{\varepsilon} u_{2}\left(x^{\prime}, 0\right) \widehat{\phi}^{\mu}\left(\frac{x}{\varepsilon}\right), \quad p_{\varepsilon}^{\mu}=p(x)-\frac{1}{\sqrt{\varepsilon}} u_{2}\left(x^{\prime}, 0\right) \widehat{q}^{\mu}\left(\frac{x}{\varepsilon}\right),
$$

then (3.18) still holds by replacing $\boldsymbol{u}$ and $p$ by $\boldsymbol{u}_{\varepsilon}^{\mu}$ and $p_{\varepsilon}^{\mu}$, respectively.

The following proposition ensures the existence and uniqueness of the solutions to systems (3.10) and (3.14), and states some exponential decay properties in the $y_{3}$-direction that will be useful to perform numerical simulations.

Proposition 3.4. There exists a unique solution $\left(\widehat{\phi}^{\lambda}, \widehat{q}^{\lambda}\right) \in \mathcal{V}^{3} \times L^{2}(\widehat{Q})$ to system (3.10). Moreover, $\left(\widehat{\phi}^{\lambda}, \widehat{q}^{\lambda}\right) \in C^{\infty}(\widehat{Q})^{3} \times C^{\infty}(\widehat{Q})$, and there exists a constant $\tau>0$ such that, for every $\alpha \geq 0$ and $t>0$, there exists a constant $C_{\alpha, t}$ such that, for every $y \in \mathcal{T}^{2} \times(0,+\infty)$,

$$
\begin{aligned}
y_{3} \geq t \Rightarrow & \left|\widehat{\phi}_{1,2}^{\lambda}(y)-\int_{\mathcal{T}^{2} \times\{0\}} \widehat{\phi}_{1,2}^{\lambda}\left(y^{\prime}, 0\right) d y^{\prime}\right|+\left|\widehat{\phi}_{3}^{\lambda}(y)\right| \leq C_{0, t} \lambda\left\|\partial_{1} \Psi_{1}\right\|_{L^{2}\left(\mathcal{T}^{1}\right)} e^{-\tau y_{3}}, \\
& y_{3} \geq t, \quad \alpha \geq 1 \Rightarrow\left|D^{\alpha} \widehat{\phi}^{\lambda}(y)\right|+\left|D^{\alpha-1} \widehat{q}^{\lambda}(y)\right| \leq C_{\alpha, t} \lambda\left\|\partial_{1} \Psi_{1}\right\|_{L^{2}\left(\mathcal{T}^{1}\right)} e^{-\tau y_{3}} .
\end{aligned}
$$

The same properties hold for $\left(\widehat{\phi}^{\mu}, \widehat{q}^{\mu}\right)$, the former inequalities being replaced by

$$
\begin{gathered}
y_{3} \geq t \Rightarrow\left|\widehat{\phi}_{1,2}^{\mu}(y)-\int_{\mathcal{T}^{2} \times\{0\}} \widehat{\phi}_{1,2}^{\mu}\left(y^{\prime}, 0\right) d y^{\prime}\right|+\left|\widehat{\phi}_{3}^{\mu}(y)\right| \\
\leq C_{0, t} \mu^{2}\left\|\partial_{1} \Psi_{1}\right\|_{L^{2}\left(\mathcal{T}^{1}\right)}\left\|\partial_{2} \Psi_{2}\right\|_{L^{2}\left(\mathcal{T}^{1}\right)} e^{-\tau y_{3}}
\end{gathered}
$$




\section{Proofs of the results}

The goal of this section is to prove Theorem 3.1. The key point of this result is to describe the asymptotic behaviour of $\left(\boldsymbol{u}_{\varepsilon}, p_{\varepsilon}\right)$ near the ribbed boundary $\Gamma_{\varepsilon}$. In order to take into account the effect of the roughness, we will apply an adaptation of the unfolding method (see e.g. [3], [11], [14], [16]) described in [15] for rough boundaries with low amplitude. Note that the unfolding method is a very efficient tool to study periodic homogenization problems where the size of the periodic cell tends to zero. The idea is to introduce suitable changes of variables which transform every periodic cell into a simpler reference set by using a supplementary variable (microscopic variable). Remark that the unfolding method is strongly related to the two-scale convergence method ( [1], [28]).

Given $\left(\boldsymbol{u}_{\varepsilon}, p_{\varepsilon}\right) \in H^{1}\left(\Omega_{\varepsilon}\right)^{3} \times L_{0}^{2}\left(\Omega_{\varepsilon}\right)$, with $\boldsymbol{u}_{\varepsilon}=0$ on $\Gamma_{\text {top }}$, we define $\left(\widehat{\boldsymbol{u}}_{\varepsilon}, \widehat{p}_{\varepsilon}\right)$ by

$$
\begin{aligned}
& \widehat{\boldsymbol{u}}_{\varepsilon}\left(x^{\prime}, y\right)=\boldsymbol{u}_{\varepsilon}\left(\varepsilon \kappa\left(\frac{x^{\prime}}{\varepsilon}\right)+\varepsilon y^{\prime}, \varepsilon y_{3}\right), \\
& \widehat{p}_{\varepsilon}\left(x^{\prime}, y\right)=p_{\varepsilon}\left(\varepsilon \kappa\left(\frac{x^{\prime}}{\varepsilon}\right)+\varepsilon y^{\prime}, \varepsilon y_{3}\right),
\end{aligned}
$$

for a.e. $\left(x^{\prime}, y^{\prime}\right) \in \mathcal{T}^{2} \times \widehat{Y}_{\varepsilon}$, with

$$
\widehat{Y}_{\varepsilon}:=\left\{y \in \mathcal{T}^{2} \times \mathbb{R}:-\Psi_{\varepsilon}\left(y^{\prime}\right)<y_{3}<\frac{1}{\varepsilon}\right\}
$$

with

$$
\Psi_{\varepsilon}\left(y^{\prime}\right):=\frac{\delta_{\varepsilon}}{\varepsilon} \Psi_{1}\left(y_{1}+\frac{\delta_{\varepsilon}}{\varepsilon} \Psi_{2}\left(y_{2}\right)\right)
$$

Remark 4.1. For $k^{\prime} \in \mathbb{Z}^{2}$ the restriction of $\left(\widehat{\boldsymbol{u}}_{\varepsilon}, \widehat{p}_{\varepsilon}\right)$ to $C_{\varepsilon}^{k^{\prime}} \times \widehat{Y}_{\varepsilon}$ does not depend on $x^{\prime}$, while as function of $y$ it is obtained from $\left(\boldsymbol{u}_{\varepsilon}, p_{\varepsilon}\right)$ by using the change of variables

$$
y^{\prime}=\frac{x^{\prime}-\varepsilon k^{\prime}}{\varepsilon}, \quad y_{3}=\frac{x_{3}}{\varepsilon},
$$

which transforms $Q_{\varepsilon}^{k^{\prime}}$ into $\widehat{Y}_{\varepsilon}$.

Lemmas 4.2 and 4.4 below describe the asymptotic behaviour of $\left(\widehat{\boldsymbol{u}}_{\varepsilon}, \widehat{p}_{\varepsilon}\right)$ in $\mathcal{T}^{2} \times \widehat{Q}_{M}$, for every $M>0$, given by (4.1), (4.2), when $\left(\boldsymbol{u}_{\varepsilon}, p_{\varepsilon}\right) \in H^{1}\left(\Omega_{\varepsilon}\right)^{3} \times L_{0}^{2}\left(\Omega_{\varepsilon}\right)$ satisfies (3.5). We remark that the proofs of Lemmas 4.2 and 4.4 are closely related to the ones described in [15]. The main difference is the presence of oscillations of the boundary along the streamwise direction, that give rise to a novel effect associated with the critical regime $\delta_{\varepsilon} \approx \varepsilon^{\frac{5}{4}}$

Lemma 4.2. Let $p_{\varepsilon}$ be in $L_{0}^{2}\left(\Omega_{\varepsilon}\right)$ with bounded norm. Then, up to a subsequence, there exists $\widehat{p} \in L^{2}\left(\mathcal{T}^{2} \times \widehat{Q}\right)$ such that the sequence $\widehat{p}_{\varepsilon}$ defined by (4.2) satisfies

$$
\sqrt{\varepsilon} \widehat{p}_{\varepsilon} \rightarrow \widehat{p} \quad \text { in } \quad L^{2}\left(\mathcal{T}^{2} \times \widehat{Q}_{M}\right) \quad \forall M>0
$$


Proof. For every $M>0$, the definition of $\widehat{p}_{\varepsilon}$ and property (3.5) yield the estimate

$$
\begin{aligned}
\int_{\mathcal{T}^{2} \times \widehat{Q}_{M}}\left|\widehat{p}_{\varepsilon}\right|^{2} d x^{\prime} d y & \leq \sum_{k^{\prime} \in I_{\varepsilon}} \varepsilon^{2} \int_{\widehat{Q}_{M}}\left|p_{\varepsilon}\left(\varepsilon\left(k^{\prime}+y^{\prime}\right), \varepsilon y_{3}\right)\right|^{2} d y \\
& \leq \frac{1}{\varepsilon} \sum_{k^{\prime} \in I_{\varepsilon}} \int_{Q_{\varepsilon}^{k^{\prime}}}\left|p_{\varepsilon}(x)\right|^{2} d x \leq \frac{1}{\varepsilon} \int_{\Omega_{\varepsilon}}\left|p_{\varepsilon}\right|^{2} d x \leq \frac{C}{\varepsilon}
\end{aligned}
$$

Thus, there exists $\widehat{p}: \mathcal{T}^{2} \times \widehat{Q} \rightarrow \mathbb{R}$ such that (4.5) holds. By semicontinuity, (4.6) implies

$$
\int_{\mathcal{T}^{2} \times \widehat{Q}_{M}}|\widehat{p}|^{2} d x^{\prime} d y \leq \liminf _{\varepsilon \rightarrow 0} \int_{\Omega_{\varepsilon}}\left|p_{\varepsilon}\right|^{2} d x, \quad \forall M>0 .
$$

We will need the following result whose proof is elementary and so, we omit it.

Lemma 4.3. Let $\boldsymbol{v}_{\varepsilon} \in L^{2}\left(\mathcal{T}^{2}\right)$ be a sequence which converges weakly to a function $\boldsymbol{v}$ in $L^{2}\left(\mathcal{T}^{2}\right)$. We define $\overline{\boldsymbol{v}}_{\varepsilon} \in L^{2}\left(\mathcal{T}^{2}\right)$ by

$$
\overline{\boldsymbol{v}}_{\varepsilon}\left(x^{\prime}\right)=\frac{1}{\varepsilon^{2}} \int_{C_{\varepsilon}\left(x^{\prime}\right)} \boldsymbol{v}_{\varepsilon}\left(z^{\prime}\right) d z^{\prime}, \text { a.e. } x^{\prime} \in \mathbb{R}^{2} .
$$

Then we have:

(i) For every $\tau^{\prime} \in \mathbb{R}^{2}$, the sequence $\frac{\overline{\boldsymbol{v}}_{\varepsilon}\left(x^{\prime}+\varepsilon \tau^{\prime}\right)-\overline{\boldsymbol{v}}_{\varepsilon}\left(x^{\prime}\right)}{\sqrt{\varepsilon}}$ converges to zero in the sense of distributions in $\mathcal{T}^{2}$.

(ii) If the convergence of $\boldsymbol{v}_{\varepsilon}$ is strong, then $\overline{\boldsymbol{v}}_{\varepsilon}$ converges strongly to $\boldsymbol{v}$ in $L^{2}\left(\mathcal{T}^{2}\right)$.

Lemma 4.4. We consider a sequence $\boldsymbol{u}_{\varepsilon} \in H^{1}\left(\Omega_{\varepsilon}\right)^{3}$ with bounded norm satisfying $\boldsymbol{u}_{\varepsilon} \cdot \boldsymbol{\nu}=$ 0 on $\Gamma_{\varepsilon}, \boldsymbol{u}_{\varepsilon}=\mathbf{0}$ on $\Gamma_{\text {top }}$, and such that (it always holds for a subsequence) there exists $\boldsymbol{u} \in H^{1}(\Omega)^{3}$ with $\boldsymbol{u}_{\varepsilon}$ converging weakly to $\boldsymbol{u}$ in $H^{1}(\Omega)^{3}$. Then, the third component $u_{3}$ of $\boldsymbol{u}$ vanishes on $\Gamma$. Moreover, we have

(i) If $\delta_{\varepsilon} \approx \varepsilon^{\frac{3}{2}}$, with $\delta_{\varepsilon} / \varepsilon^{\frac{3}{2}} \rightarrow \lambda, 0<\lambda<+\infty$, then there exists $\widehat{\boldsymbol{u}} \in L^{2}\left(\mathcal{T}^{2} ; \mathcal{V}^{3}\right)$ with

$$
\widehat{u}_{3}\left(x^{\prime}, y^{\prime}, 0\right)=-\lambda \partial_{y_{1}} \Psi_{1}\left(y_{1}\right) u_{1}\left(x^{\prime}, 0\right), \text { a.e. }\left(x^{\prime}, y^{\prime}\right) \in \mathcal{T}^{2} \times \mathcal{T}^{2},
$$

such that for every $M>0$, the sequence $\widehat{\boldsymbol{u}}_{\varepsilon}$ defined by (4.1) satisfies

$$
\frac{1}{\sqrt{\varepsilon}} D_{y} \widehat{\boldsymbol{u}}_{\varepsilon} \rightarrow D_{y} \widehat{\boldsymbol{u}} \text { in } L^{2}\left(\mathcal{T}^{2} \times \widehat{Q}_{M}\right)^{3 \times 3} .
$$

Besides, if $\operatorname{div} \boldsymbol{u}_{\varepsilon}=0$ in $\Omega_{\varepsilon}$, then

$$
\operatorname{div}_{y} \widehat{\boldsymbol{u}}=0 \text { in } \mathcal{T}^{2} \times \widehat{Q} .
$$

(ii) If $\varepsilon^{\frac{3}{2}} \ll \delta_{\varepsilon} \ll \varepsilon^{\frac{5}{4}}$, then

$$
u_{1}\left(x^{\prime}, 0\right) \partial_{y_{1}} \Psi_{1}\left(y_{1}\right)=0 \quad \text { a.e. }\left(x^{\prime}, y^{\prime}\right) \in \mathcal{T}^{2} \times \mathcal{T}^{2} .
$$


(iii) If $\delta_{\varepsilon} \approx \varepsilon^{\frac{5}{4}}$, with $\delta_{\varepsilon} / \varepsilon^{\frac{5}{4}} \rightarrow \mu, 0<\mu<+\infty$, then $u_{1}$ satisfies (4.10), and there exists $\widehat{\boldsymbol{u}} \in L^{2}\left(\mathcal{T}^{2} ; \mathcal{V}^{3}\right)$ with

$$
\widehat{u}_{3}\left(x^{\prime}, y^{\prime}, 0\right)=-\mu^{2} \partial_{y_{1}} \Psi_{1}\left(y_{1}\right) \partial_{y_{2}} \Psi_{2}\left(y_{2}\right) u_{2}\left(x^{\prime}, 0\right), \quad \text { a.e. }\left(x^{\prime}, y^{\prime}\right) \in \mathcal{T}^{2} \times \mathcal{T}^{2},
$$

such that the sequence $\widehat{\boldsymbol{u}}_{\varepsilon}$ satisfies (4.8), and moreover (4.9) also holds if $\operatorname{div} \boldsymbol{u}_{\varepsilon}=0$ in $\Omega_{\varepsilon}$.

(iv) If $\delta_{\varepsilon} \gg \varepsilon^{\frac{5}{4}}$, then $u_{1}$ satisfies (4.10), and $u_{2}$ satisfies

$$
u_{2}\left(x^{\prime}, 0\right) \partial_{1} \Psi_{1}\left(y_{1}\right) \partial_{2} \Psi_{2}\left(y_{2}\right)=0 \text { a.e. }\left(x^{\prime}, y^{\prime}\right) \in \mathcal{T}^{2} \times \mathcal{T}^{2}
$$

Proof. We divide the proof in five steps.

Step 1 Let us first prove that $u_{3}$ vanishes on $\Gamma$.

Since $\boldsymbol{u}_{\varepsilon} \cdot \boldsymbol{\nu}=0$ on $\Gamma_{\varepsilon}$, for every $\varphi \in C_{c}^{1}\left(\mathcal{T}^{2} \times(-1,1)\right)$ we have

$$
\int_{\Omega_{\varepsilon}} \boldsymbol{u}_{\varepsilon} \nabla \varphi d x=-\int_{\Omega_{\varepsilon}} \operatorname{div} \boldsymbol{u}_{\varepsilon} \varphi d x
$$

Using

$$
\begin{gathered}
\left|\int_{\Omega_{\varepsilon} \backslash \Omega} \boldsymbol{u}_{\varepsilon} \nabla \varphi d x\right| \leq\left(\int_{\Omega_{\varepsilon}}\left|\boldsymbol{u}_{\varepsilon}\right|^{2} d x\right)^{\frac{1}{p}}\left(\int_{\Omega_{\varepsilon} \backslash \Omega}|\nabla \varphi|^{2} d x\right)^{\frac{1}{2}} \rightarrow 0, \\
\left|\int_{\Omega_{\varepsilon} \backslash \Omega} \operatorname{div} \boldsymbol{u}_{\varepsilon} \varphi d x\right| \leq\left(\int_{\Omega_{\varepsilon}}\left|\operatorname{div} \boldsymbol{u}_{\varepsilon}\right|^{2} d x\right)^{\frac{1}{2}}\left(\int_{\Omega_{\varepsilon} \backslash \Omega}|\varphi|^{2} d x\right)^{\frac{1}{2}} \rightarrow 0,
\end{gathered}
$$

and the weak convergence of $\boldsymbol{u}_{\varepsilon}$ to $\boldsymbol{u}$ in $H^{1}(\Omega)^{3}$, we can pass to the limit in (4.13) to deduce

$$
\int_{\Omega} \boldsymbol{u} \nabla \varphi d x=-\int_{\Omega} \operatorname{div} \boldsymbol{u} \varphi d x
$$

and then

$$
\int_{\Gamma} u_{3} \varphi d x^{\prime}=0, \quad \forall \varphi \in C_{c}^{1}\left(\mathcal{T}^{2} \times(-1,1)\right),
$$

which proves $u_{3}=0$ on $\Gamma$.

Step 2 Let us obtain some estimates for the sequence $\widehat{\boldsymbol{u}}_{\varepsilon}$ given by (4.1).

For $M>0$, the definition (4.1) of $\widehat{\boldsymbol{u}}_{\varepsilon}$ proves for every $\varepsilon>0$ small enough

$$
\begin{aligned}
\int_{\mathcal{T}^{2} \times \widehat{Q}_{M}}\left|D_{y} \widehat{\boldsymbol{u}}_{\varepsilon}\left(x^{\prime}, y\right)\right|^{2} d x^{\prime} d y & \leq \varepsilon^{4} \sum_{k^{\prime} \in I_{\varepsilon}} \int_{\widehat{Q}_{M}}\left|D \boldsymbol{u}_{\varepsilon}\left(\varepsilon\left(k^{\prime}+y^{\prime}\right), \varepsilon y_{3}\right)\right|^{2} d y \\
& \leq \sum_{k^{\prime} \in I_{\varepsilon}} \varepsilon \int_{Q_{\varepsilon}^{k^{\prime}}}\left|D \boldsymbol{u}_{\varepsilon}\right|^{2} d x \leq \varepsilon \int_{\Omega_{\varepsilon}}\left|D \boldsymbol{u}_{\varepsilon}\right|^{2} d x \leq C \varepsilon .
\end{aligned}
$$

On the other hand, defining

$$
\overline{\boldsymbol{u}}_{\varepsilon}\left(x^{\prime}\right)=\frac{1}{\varepsilon^{2}} \int_{C_{\varepsilon}\left(x^{\prime}\right)} \boldsymbol{u}_{\varepsilon}\left(\tau^{\prime}, 0\right) d \tau=\int_{\mathcal{T}^{2}} \widehat{\boldsymbol{u}}_{\varepsilon}\left(x^{\prime}, y^{\prime}, 0\right) d y^{\prime},
$$


and using the inequality

$$
\int_{\widehat{Q}_{M}}\left|\widehat{\boldsymbol{u}}_{\varepsilon}\left(x^{\prime}, y\right)-\overline{\boldsymbol{u}}_{\varepsilon}\left(x^{\prime}\right)\right|^{2} d y \leq C_{M} \int_{\widehat{Q}_{M}}\left|D_{y} \widehat{\boldsymbol{u}}_{\varepsilon}\right|^{2} d y, \text { a.e. } x^{\prime} \in \mathbb{R}^{2},
$$

where $C_{M}$ does not depend on $\varepsilon$ and taking into account (4.14), we deduce that

$$
\widehat{\boldsymbol{U}}_{\varepsilon}=\frac{\widehat{\boldsymbol{u}}_{\varepsilon}\left(x^{\prime}, y\right)-\overline{\boldsymbol{u}}_{\varepsilon}}{\sqrt{\varepsilon}} \text { is bounded in } L^{2}\left(\mathcal{T}^{2} ; H^{1}\left(\widehat{Q}_{M}\right)^{3}\right) \quad \forall M>0 .
$$

Thus, there exists $\widehat{\boldsymbol{u}}: \mathcal{T}^{2} \times \widehat{Q} \rightarrow \mathbb{R}^{3}$, such that, up to a subsequence,

$$
\widehat{\boldsymbol{U}}_{\varepsilon} \rightarrow \widehat{\boldsymbol{u}} \text { in } L^{2}\left(\mathcal{T}^{2} ; H^{1}\left(\widehat{Q}_{M}\right)^{3}\right) \quad \forall M>0,
$$

and then

$$
\frac{1}{\sqrt{\varepsilon}} D_{y} \widehat{\boldsymbol{u}}_{\varepsilon} \rightarrow D_{y} \widehat{\boldsymbol{u}} \text { in } L^{2}\left(\mathcal{T}^{2} \times Q_{M}\right)^{3 \times 3} \quad \forall M>0 .
$$

Passing to the limit by semicontinuity in inequalities (4.14) and (4.16) (this latest one after integration in $\mathcal{T}^{2}$ ), we get

$$
\int_{\mathcal{T}^{2} \times \widehat{Q}_{M}}\left|D_{y} \widehat{\boldsymbol{u}}\right|^{2} d x^{\prime} d y \leq C, \quad \int_{\mathcal{T}^{2} \times \widehat{Q}_{M}}|\widehat{\boldsymbol{u}}|^{2} d x^{\prime} d y \leq C_{M},
$$

and by the arbitrariness of $M$, once we prove the $\mathcal{T}^{2}$-periodicity of $\widehat{\boldsymbol{u}}$ in $y^{\prime}$ (Step 3), then

$$
\widehat{\boldsymbol{u}} \in L^{2}\left(\mathcal{T}^{2} ; \mathcal{V}^{3}\right)
$$

Moreover, if we also assume that $\operatorname{div} \boldsymbol{u}_{\varepsilon}=0$ in $\Omega_{\varepsilon}$, then by definition (4.1) of $\widehat{\boldsymbol{u}}_{\varepsilon}$, we have $\operatorname{div}_{y} \widehat{\boldsymbol{u}}_{\varepsilon}=0$ in $\mathcal{T}^{2} \times \widehat{Q}_{M}$, which together with (4.19) proves

$$
\operatorname{div}_{y} \widehat{\boldsymbol{u}}=0 \text { in } \mathcal{T}^{2} \times \widehat{Q} .
$$

Step 3 Let us prove that $\widehat{\boldsymbol{u}}$ is $\mathcal{T}^{2}$-periodic in $y^{\prime}$.

We observe that by definition (4.1) of $\widehat{\boldsymbol{u}}_{\varepsilon}$, for every $M>0$, we have

$$
\widehat{\boldsymbol{u}}_{\varepsilon}\left(x_{1}+\varepsilon, x_{2}, 0, y_{2}, y_{3}\right)=\widehat{\boldsymbol{u}}_{\varepsilon}\left(x^{\prime}, 1, y_{2}, y_{3}\right) \text { a.e. }\left(x^{\prime}, y_{2}, y_{3}\right) \in \mathcal{T}^{2} \times \mathcal{T}^{1} \times(0, M) .
$$

Therefore the sequence $\widehat{\boldsymbol{U}}_{\varepsilon}$ satisfies

$$
\widehat{\boldsymbol{U}}_{\varepsilon}\left(x_{1}+\varepsilon, x_{2}, 0, y_{2}, y_{3}\right)-\widehat{\boldsymbol{U}}_{\varepsilon}\left(x^{\prime}, 1, y_{2}, y_{3}\right)=\frac{-\overline{\boldsymbol{u}}_{\varepsilon}\left(x_{1}+\varepsilon, x_{2}\right)+\overline{\boldsymbol{u}}_{\varepsilon}\left(x^{\prime}\right)}{\sqrt{\varepsilon}} .
$$

By (4.15) and the fact that $\boldsymbol{u}_{\varepsilon \mid \Gamma}$ is bounded in $L^{2}(\Gamma)^{3}$, we can apply Lemma 4.3(i) to deduce that the right-hand side of this equality tends to zero in the sense of distributions in $\mathcal{T}^{2}$. Therefore, passing to the limit in (4.22) by (4.18), and taking into account the arbitrariness of $M$ we get

$$
\widehat{\boldsymbol{u}}\left(x^{\prime}, 0, y_{2}, y_{3}\right)-\widehat{\boldsymbol{u}}\left(x^{\prime}, 1, y_{2}, y_{3}\right)=0 \text { a.e. }\left(x^{\prime}, y_{2}, y_{3}\right) \in \mathcal{T}^{2} \times \mathcal{T}^{1} \times \mathbb{R} .
$$

Analogously, we can prove

$$
\widehat{\boldsymbol{u}}\left(x^{\prime}, y_{1}, 0, y_{3}\right)-\widehat{\boldsymbol{u}}\left(x^{\prime}, y_{1}, 1, y_{3}\right)=0 \text { a.e. }\left(x^{\prime}, y_{1}, y_{3}\right) \in \mathcal{T}^{2} \times \mathcal{T}^{1} \times \mathbb{R} .
$$

These equalities prove that $\widehat{\boldsymbol{u}}$ is periodic with respect to $\mathcal{T}^{2}$. 
Step 4 Using the compact embedding of $H^{1}(\Omega)$ into $L^{2}(\Gamma)$ and Lemma 4.3-(ii), we have that $\overline{\boldsymbol{u}}_{\varepsilon}$ converges strongly to $\boldsymbol{u}\left(x^{\prime}, 0\right)$ in $L^{2}\left(\mathcal{T}^{2}\right)^{3}$. Thus, by (4.17), we deduce

$$
\widehat{\boldsymbol{u}}_{\varepsilon}\left(x^{\prime}, y\right) \rightarrow \boldsymbol{u}\left(x^{\prime}, 0\right) \text { in } L^{2}\left(\mathcal{T}^{2} ; H^{1}\left(\widehat{Q}_{M}\right)^{3}\right) \quad \forall M>0
$$

Step 5 Using the change of variables (4.4), which defines $\widehat{\boldsymbol{u}}_{\varepsilon}$, in the equality $\boldsymbol{u}_{\varepsilon} \cdot \boldsymbol{\nu}=0$ on $\Gamma_{\varepsilon}$, we get

$$
\begin{aligned}
& \partial_{y_{1}} \Psi_{\varepsilon}\left(y^{\prime}\right) \widehat{\boldsymbol{u}}_{\varepsilon, 1}\left(x^{\prime}, y^{\prime},-\Psi_{\varepsilon}\left(y^{\prime}\right)\right)+\partial_{y_{2}} \Psi_{\varepsilon}\left(y^{\prime}\right) \widehat{\boldsymbol{u}}_{\varepsilon, 2}\left(x^{\prime}, y^{\prime},-\Psi_{\varepsilon}\left(y^{\prime}\right)\right) \\
&+\widehat{\boldsymbol{u}}_{\varepsilon, 3}\left(x^{\prime}, y^{\prime},-\Psi_{\varepsilon}\left(y^{\prime}\right)\right)=0
\end{aligned}
$$

a.e. in $\mathcal{T}^{2} \times \mathcal{T}^{2}$, where, taking into account the definition of $\Psi_{\varepsilon}\left(y^{\prime}\right)$ given by (4.3)

$$
\begin{aligned}
& \partial_{y_{1}} \Psi_{\varepsilon}\left(y^{\prime}\right)=\frac{\delta_{\varepsilon}}{\varepsilon} \partial_{y_{1}} \Psi_{1}\left(y_{1}+\frac{\delta_{\varepsilon}}{\varepsilon} \Psi_{2}\left(y_{2}\right)\right) \\
& \partial_{y_{2}} \Psi_{\varepsilon}\left(y^{\prime}\right)=\frac{\delta_{\varepsilon}^{2}}{\varepsilon^{2}} \partial_{y_{1}} \Psi_{1}\left(y_{1}+\frac{\delta_{\varepsilon}}{\varepsilon} \Psi_{2}\left(y_{2}\right)\right) \partial_{y_{2}} \Psi_{2}\left(y_{2}\right) .
\end{aligned}
$$

Thanks to (4.24) and (4.14), we have then

$$
\begin{aligned}
& \left|\partial_{y_{1}} \Psi_{\varepsilon}\left(y^{\prime}\right) \widehat{u}_{\varepsilon, 1}\left(x^{\prime}, y^{\prime}, 0\right)+\partial_{y_{2}} \Psi_{\varepsilon}\left(y^{\prime}\right) \widehat{u}_{\varepsilon, 2}\left(x^{\prime}, y^{\prime}, 0\right)+\widehat{u}_{\varepsilon, 3}\left(x^{\prime}, y^{\prime}, 0\right)\right| \\
& \leq \int_{-\Psi_{\varepsilon}\left(y^{\prime}\right)}^{0}\left|\partial_{y_{1}} \Psi_{\varepsilon}\left(y^{\prime}\right) \partial_{3} \widehat{u}_{\varepsilon, 1}\left(x^{\prime}, y^{\prime}, t\right)+\partial_{y_{2}} \Psi_{\varepsilon}\left(y^{\prime}\right) \partial_{3} \widehat{u}_{\varepsilon, 2}\left(x^{\prime}, y^{\prime}, t\right)+\partial_{3} \widehat{u}_{\varepsilon, 3}\left(x^{\prime}, y^{\prime}, t\right)\right| d t \\
& \leq C\left(\frac{\delta_{\varepsilon}}{\varepsilon}\right)^{\frac{1}{2}}\left(\int_{-\Psi_{\varepsilon}\left(y^{\prime}\right)}^{0}\left|\partial_{y_{3}} \widehat{\boldsymbol{u}}_{\varepsilon}\left(x^{\prime}, y^{\prime}, t\right)\right|^{2} d t\right)^{\frac{1}{2}} \text { a.e. }\left(x^{\prime}, y^{\prime}\right) \in \mathcal{T}^{2} \times \mathcal{T}^{2} .
\end{aligned}
$$

Taking the power 2, integrating in $\mathcal{T}^{2} \times \mathcal{T}^{2}$ and using (4.14) we then deduce

$$
\int_{\mathcal{T}^{2} \times \mathcal{T}^{2}}\left|\partial_{y_{1}} \Psi_{\varepsilon}\left(y^{\prime}\right) \widehat{u}_{\varepsilon, 1}\left(x^{\prime}, y^{\prime}, 0\right)+\partial_{y_{2}} \Psi_{\varepsilon}\left(y^{\prime}\right) \widehat{u}_{\varepsilon, 2}\left(x^{\prime}, y^{\prime}, 0\right)+\widehat{u}_{\varepsilon, 3}\left(x^{\prime}, y^{\prime}, 0\right)\right|^{2} d x^{\prime} d y^{\prime} \leq C \delta_{\varepsilon},
$$

which implies

$$
\begin{aligned}
& \int_{\mathcal{T}^{2} \times \mathcal{T}^{2}} \mid \partial_{y_{1}} \Psi_{\varepsilon}\left(y^{\prime}\right) \widehat{u}_{\varepsilon, 1}\left(x^{\prime}, y^{\prime}, 0\right)+\partial_{y_{2}} \Psi_{\varepsilon}\left(y^{\prime}\right) \widehat{u}_{\varepsilon, 2}\left(x^{\prime}, y^{\prime}, 0\right)+\widehat{u}_{\varepsilon, 3}\left(x^{\prime}, y^{\prime}, 0\right) \\
& -\left.\int_{\mathcal{T}^{2}}\left(\partial_{y_{1}} \Psi_{\varepsilon}\left(z^{\prime}\right) \widehat{u}_{\varepsilon, 1}\left(x^{\prime}, z^{\prime}, 0\right)+\partial_{y_{2}} \Psi_{\varepsilon}\left(z^{\prime}\right) \widehat{u}_{\varepsilon, 2}\left(x^{\prime}, z^{\prime}, 0\right)+\widehat{u}_{\varepsilon, 3}\left(x^{\prime}, z^{\prime}, 0\right)\right) d z^{\prime}\right|^{2} d x^{\prime} d y^{\prime} \leq C \delta_{\varepsilon} .
\end{aligned}
$$

Dividing by $\varepsilon$, and taking into account that both $\partial_{y_{1}} \Psi_{\varepsilon}, \partial_{y_{2}} \Psi_{\varepsilon}$ have mean value zero 
in $\mathcal{T}^{2}$, we get

$$
\begin{aligned}
\int_{\mathcal{T}^{2} \times \mathcal{T}^{2}} & \mid \frac{\delta_{\varepsilon}}{\varepsilon^{\frac{3}{2}}} \partial_{y_{1}} \Psi_{1}\left(y_{1}+\frac{\delta_{\varepsilon}}{\varepsilon} \Psi_{2}\left(y_{2}\right)\right) \widehat{u}_{\varepsilon, 1}\left(x^{\prime}, y^{\prime}, 0\right) \\
& -\frac{\delta_{\varepsilon}}{\varepsilon} \int_{\mathcal{T}^{2}} \partial_{y_{1}} \Psi_{1}\left(y_{1}+\frac{\delta_{\varepsilon}}{\varepsilon} \Psi_{2}\left(y_{2}\right)\right)\left(\frac{\widehat{u}_{\varepsilon, 1}\left(x^{\prime}, z^{\prime}, 0\right)-\bar{u}_{\varepsilon, 1}\left(x^{\prime}\right)}{\sqrt{\varepsilon}}\right) d z^{\prime} \\
& +\frac{\delta_{\varepsilon}^{2}}{\varepsilon^{\frac{5}{2}}} \partial_{y_{1}} \Psi_{1}\left(y_{1}+\frac{\delta_{\varepsilon}}{\varepsilon} \Psi_{2}\left(y_{2}\right)\right) \partial_{y_{2}} \Psi_{2}\left(y_{2}\right) \widehat{u}_{\varepsilon, 2}\left(x^{\prime}, y^{\prime}, 0\right) \\
& -\frac{\delta_{\varepsilon}^{2}}{\varepsilon^{2}} \int_{\mathcal{T}^{2}} \partial_{y_{1}} \Psi_{1}\left(y_{1}+\frac{\delta_{\varepsilon}}{\varepsilon} \Psi_{2}\left(y_{2}\right)\right) \partial_{y_{2}} \Psi_{2}\left(y_{2}\right)\left(\frac{\widehat{u}_{\varepsilon, 2}\left(x^{\prime}, z^{\prime}, 0\right)-\bar{u}_{\varepsilon, 2}\left(x^{\prime}\right)}{\sqrt{\varepsilon}}\right) d z^{\prime} \\
& +\left.\frac{\widehat{u}_{\varepsilon, 3}\left(x^{\prime}, y^{\prime}, 0\right)-\bar{u}_{\varepsilon, 3}\left(x^{\prime}\right)}{\sqrt{\varepsilon}}\right|^{2} d x^{\prime} d y^{\prime} \leq C \frac{\delta_{\varepsilon}}{\varepsilon} \rightarrow 0 .
\end{aligned}
$$

Depending on the values of $\lambda$ and $\mu$, limits of $\delta_{\varepsilon} / \varepsilon^{\frac{3}{2}}$ and $\delta_{\varepsilon} / \varepsilon^{\frac{5}{4}}$ respectively, we deduce:

- When $\delta_{\varepsilon} \approx \varepsilon^{\frac{3}{2}}$, then we know that $\frac{\delta_{\varepsilon}}{\varepsilon^{\frac{3}{2}}} \partial_{y_{1}} \Psi_{1}\left(y_{1}+\frac{\delta_{\varepsilon}}{\varepsilon} \Psi_{2}\left(y_{2}\right)\right) \widehat{u}_{\varepsilon, 1}\left(x^{\prime}, y^{\prime}, 0\right)$ is bounded in $L^{2}\left(\mathcal{T}^{2} \times \mathcal{T}^{2}\right)$ and we can pass to the limit by using (4.23) for stating

$$
\partial_{y_{1}} \Psi_{1}\left(y_{1}+\frac{\delta_{\varepsilon}}{\varepsilon} \Psi_{2}\left(y_{2}\right)\right) \widehat{u}_{\varepsilon, 1}\left(x^{\prime}, y^{\prime}, 0\right) \rightarrow \partial_{y_{1}} \Psi_{1}\left(y_{1}\right) u_{1}\left(x^{\prime}, 0\right) \text { in } L^{2}\left(\mathcal{T}^{2} \times \mathcal{T}^{2}\right) .
$$

This gives with (4.25) and (4.18),

$$
\widehat{u}_{3}\left(x^{\prime}, y^{\prime}, 0\right)=-\lambda \partial_{y_{1}} \Psi_{1}\left(y_{1}\right) u_{1}\left(x^{\prime}, 0\right) \text { a.e. }\left(x^{\prime}, y^{\prime}\right) \in \mathcal{T}^{2} \times \mathcal{T}^{2} .
$$

- When $\delta_{\varepsilon} \gg \varepsilon^{\frac{3}{2}}$, then, because we impose $\delta_{\varepsilon} / \varepsilon \rightarrow 0,(4.25)$ and (4.17) imply

$$
\partial_{y_{1}} \Psi_{1}\left(y_{1}+\frac{\delta_{\varepsilon}}{\varepsilon} \Psi_{2}\left(y_{2}\right)\right) \widehat{u}_{\varepsilon, 1}\left(x^{\prime}, y^{\prime}, 0\right) \rightarrow 0 \text { in } L^{2}\left(\mathcal{T}^{2} \times \mathcal{T}^{2}\right)
$$

From (4.26) and (4.23), we deduce

$$
u_{1}\left(x^{\prime}, 0\right) \partial_{y_{1}} \Psi_{1}\left(y_{1}\right)=0 \quad \text { a.e. }\left(x^{\prime}, y^{\prime}\right) \in \mathcal{T}^{2} \times \mathcal{T}^{2} .
$$

- When $\delta_{\varepsilon} \approx \varepsilon^{\frac{5}{4}}$, then we know that $\frac{\delta_{\varepsilon}^{2}}{\varepsilon^{\frac{5}{2}}} \partial_{y_{1}} \Psi_{1}\left(y_{1}+\frac{\delta_{\varepsilon}}{\varepsilon} \Psi_{2}\left(y_{2}\right)\right) \partial_{y_{2}} \Psi_{2}\left(y_{2}\right) \widehat{u}_{\varepsilon, 2}\left(x^{\prime}, y^{\prime}, 0\right)$ is bounded in $L^{2}\left(\mathcal{T}^{2} \times \mathcal{T}^{2}\right)$ and we can pass to the limit by using (4.23) for stating

$\partial_{y_{1}} \Psi_{1}\left(y_{1}+\frac{\delta_{\varepsilon}}{\varepsilon} \Psi_{2}\left(y_{2}\right)\right) \partial_{y_{2}} \Psi_{2}\left(y_{2}\right) \widehat{u}_{\varepsilon, 2}\left(x^{\prime}, y^{\prime}, 0\right) \rightarrow \partial_{y_{1}} \Psi_{1}\left(y_{1}\right) \partial_{y_{2}} \Psi_{2}\left(y_{2}\right) u_{2}\left(x^{\prime}, 0\right)$ in $L^{2}\left(\mathcal{T}^{2} \times \mathcal{T}^{2}\right)$

This gives with (4.25) and (4.18),

$$
\widehat{u}_{3}\left(x^{\prime}, y^{\prime}, 0\right)=-\mu^{2} \partial_{y_{1}} \Psi_{1}\left(y_{1}\right) \partial_{y_{2}} \Psi_{2}\left(y_{2}\right) u_{2}\left(x^{\prime}, 0\right) \text { a.e. }\left(x^{\prime}, y^{\prime}\right) \in \mathcal{T}^{2} \times \mathcal{T}^{2} \text {. }
$$


- When $\delta_{\varepsilon} \gg \varepsilon^{\frac{5}{4}}$, then, because we impose $\delta_{\varepsilon} / \varepsilon \rightarrow 0$, (4.25) and (4.17) imply

$$
\partial_{y_{1}} \Psi_{1}\left(y_{1}+\frac{\delta_{\varepsilon}}{\varepsilon} \Psi_{2}\left(y_{2}\right)\right) \partial_{y_{2}} \Psi_{2}\left(y_{2}\right) \widehat{u}_{\varepsilon, 2}\left(x^{\prime}, y^{\prime}, 0\right) \rightarrow 0 \text { in } L^{2}\left(\mathcal{T}^{2} \times \mathcal{T}^{2}\right) .
$$

From (4.27) and (4.23), we deduce

$$
u_{2}\left(x^{\prime}, 0\right) \partial_{1} \Psi_{1}\left(y_{1}\right) \partial_{2} \Psi_{2}\left(y_{2}\right)=0 \text { a.e. }\left(x^{\prime}, y^{\prime}\right) \in \mathcal{T}^{2} \times \mathcal{T}^{2} .
$$

Finally, using these properties, we easily deduce the statements of Lemma 4.4.

Proof of Theorem 3.1. Thanks to (3.5), there exists a subsequence of $\varepsilon$, still denoted by $\varepsilon$, and $(\boldsymbol{u}, p) \in H^{1}(\Omega)^{3} \times L^{2}(\Omega)$ such that (3.6) holds.

On the other hand, we observe that $\left(\boldsymbol{u}_{\varepsilon}, p_{\varepsilon}\right)$ satisfies the variational equation

$$
\left\{\begin{array}{c}
\int_{\Omega_{\varepsilon}} D \boldsymbol{u}_{\varepsilon}: D \boldsymbol{v}_{\varepsilon}-\int_{\Omega_{\varepsilon}} p_{\varepsilon} \operatorname{div} \boldsymbol{v}_{\varepsilon} d x=\int_{\Omega_{\varepsilon}} \boldsymbol{f} \cdot \boldsymbol{v}_{\varepsilon} d x \\
\forall \boldsymbol{v}_{\varepsilon} \in H^{1}\left(\Omega_{\varepsilon}\right)^{3}, \quad \boldsymbol{v}_{\varepsilon} \cdot \boldsymbol{\nu}=0 \text { on } \Gamma_{\varepsilon}, \quad \boldsymbol{v}_{\varepsilon}=\mathbf{0} \text { on } \Gamma_{\mathrm{top}} .
\end{array}\right.
$$

The proof of Theorem 3.1 is similar to the one given in [15], and it will be carried out using suitable test functions $\boldsymbol{v}_{\varepsilon}$ depending on the values of $\delta_{\varepsilon}$. In particular, the cases $(i)-($ iii $)$ have already been addressed in [15]. Here, we will develop in more detail the new critical case $(i v)$, that is when $\delta_{\varepsilon} \approx \varepsilon^{\frac{5}{4}}$, with $\delta_{\varepsilon} / \varepsilon^{\frac{5}{4}} \rightarrow \mu, 0<\mu<+\infty$. Finally, case $(v)$ will be described briefly.

Step 1 Case $\delta_{\varepsilon} \approx \varepsilon^{\frac{5}{4}}$, with $\delta_{\varepsilon} / \varepsilon^{\frac{5}{4}} \rightarrow \mu, 0<\mu<+\infty$.

We consider $\boldsymbol{v} \in C_{c}^{1}\left(\mathcal{T}^{2} \times(-1,1)\right)^{3}$ and $\widehat{\boldsymbol{v}} \in C_{c}^{1}\left(\mathcal{T}^{2} ; C(\widehat{Q})^{3}\right)$, with $D_{y} \widehat{\boldsymbol{v}}\left(x^{\prime}, y\right)=0$ a.e. in $\left\{y_{3}>M\right\}$, for some $M>0$, such that

$$
\begin{gathered}
\left\{\begin{array}{rr}
\boldsymbol{v}(x)=\boldsymbol{v}\left(x^{\prime}, 0\right) & \text { if } x_{3} \leq 0, \\
v_{3}\left(x^{\prime}, 0\right)=0, & \text { a.e. }\left(x^{\prime}, y^{\prime}\right) \in \mathcal{T}^{2} \times \mathcal{T}^{2}, \\
v_{1}\left(x^{\prime}, 0\right) \partial_{y_{1}} \Psi_{1}\left(y_{1}\right)=0 & \text { if } y_{3} \leq 0,
\end{array}\right. \\
\left\{\begin{array}{cc}
\widehat{\boldsymbol{v}}\left(x^{\prime}, y\right)=\widehat{\boldsymbol{v}}\left(x^{\prime}, y^{\prime}, 0\right) & \\
\widehat{v}_{3}\left(x^{\prime}, y^{\prime}, 0\right)=-\mu^{2} \partial_{y_{1}} \Psi_{1}\left(y_{1}\right) \partial_{2} \Psi_{2}\left(y_{2}\right) v_{2}\left(x^{\prime}, 0\right) . &
\end{array}\right.
\end{gathered}
$$

Besides, we take $\zeta \in C^{\infty}(\mathbb{R})$ such that

$$
\zeta\left(x_{3}\right)=\left\{\begin{array}{c}
1 \text { if } x_{3}<\frac{1}{3}, \\
0 \text { if } x_{3}>\frac{2}{3}
\end{array}\right.
$$

and $R_{\varepsilon}>0$ such that

$$
R_{\varepsilon} \rightarrow+\infty, \quad R_{\varepsilon}\left[\left(\frac{\delta_{\varepsilon}^{2}}{\varepsilon^{\frac{5}{2}}}-\mu^{2}\right)^{2}+\frac{\delta_{\varepsilon}^{2}}{\varepsilon^{2}}\right] \rightarrow 0 .
$$


Then, we define $\boldsymbol{v}_{\varepsilon} \in H^{1}\left(\Omega_{\varepsilon}\right)^{3}$ by

$$
\left\{\begin{array}{c}
v_{\varepsilon, 1}(x)=v_{1}(x)+\sqrt{\varepsilon} \widehat{v}_{1}\left(x^{\prime}, \frac{x}{\varepsilon}\right) \zeta\left(x_{3}\right) \\
v_{\varepsilon, 2}(x)=v_{2}(x)+\sqrt{\varepsilon} \widehat{v}_{2}\left(x^{\prime}, \frac{x}{\varepsilon}\right) \zeta\left(x_{3}\right) \\
v_{\varepsilon, 3}(x)=v_{3}(x)+\sqrt{\varepsilon}\left[\widehat{v}_{3}\left(x^{\prime}, \frac{x}{\varepsilon}\right) \zeta\left(x_{3}\right)+\right. \\
+\mu^{2} \zeta\left(\frac{x_{3}}{\varepsilon R_{\varepsilon}}\right) \partial_{1} \Psi_{1}\left(\frac{x_{1}}{\varepsilon}\right) \partial_{2} \Psi_{2}\left(\frac{x_{2}}{\varepsilon}\right) v_{2}\left(x^{\prime}, 0\right) \\
-\frac{\delta_{\varepsilon}^{2}}{\varepsilon^{\frac{5}{2}}} \zeta\left(\frac{x_{3}}{\varepsilon R_{\varepsilon}}\right) \partial_{1} \Psi_{1}\left(\frac{x_{1}}{\varepsilon}+\frac{\delta_{\varepsilon}}{\varepsilon} \Psi_{2}\left(\frac{x_{2}}{\varepsilon}\right)\right) \partial_{2} \Psi_{2}\left(\frac{x_{2}}{\varepsilon}\right) v_{2}\left(x^{\prime}, 0\right) \\
\left.-\frac{\delta_{\varepsilon}^{2}}{\varepsilon^{2}} \zeta\left(\frac{x_{3}}{\varepsilon R_{\varepsilon}}\right) \partial_{1} \Psi_{1}\left(\frac{x_{1}}{\varepsilon}+\frac{\delta_{\varepsilon}}{\varepsilon} \Psi_{2}\left(\frac{x_{2}}{\varepsilon}\right)\right) \partial_{2} \Psi_{2}\left(\frac{x_{2}}{\varepsilon}\right) \widehat{v}_{2}\left(x^{\prime}, \frac{x^{\prime}}{\varepsilon}, 0\right)\right] \\
-\frac{\delta_{\varepsilon}}{\varepsilon} \zeta\left(\frac{x_{3}}{\varepsilon^{2} R_{\varepsilon}}\right) \partial_{1} \Psi_{1}\left(\frac{x_{1}}{\varepsilon}+\frac{\delta_{\varepsilon}}{\varepsilon} \Psi_{2}\left(\frac{x_{2}}{\varepsilon}\right)\right) v_{1}\left(x^{\prime}, 0\right) \\
-\frac{\delta_{\varepsilon}}{\sqrt{\varepsilon}} \zeta\left(\frac{x_{3}}{\varepsilon R_{\varepsilon}}\right) \partial_{1} \Psi_{1}\left(\frac{x_{1}}{\varepsilon}+\frac{\delta_{\varepsilon}}{\varepsilon} \Psi_{2}\left(\frac{x_{2}}{\varepsilon}\right)\right) \widehat{v}_{1}\left(x^{\prime}, \frac{x^{\prime}}{\varepsilon}, 0\right) .
\end{array}\right.
$$

Taking into account properties given in (4.29), the sequence $\boldsymbol{v}_{\varepsilon}$ satisfies

$$
\boldsymbol{v}_{\varepsilon}=0 \text { on } \Gamma_{\text {top }}, \quad \boldsymbol{v}_{\varepsilon} \cdot \boldsymbol{\nu}=0 \text { on } \Gamma_{\varepsilon} .
$$

Thus, we can take such $\boldsymbol{v}_{\varepsilon}$ as test function in (4.28). The problem is to pass to the limit in every term that appears in (4.28). To simplify the computations, we will first estimate the derivatives of $\boldsymbol{v}_{\varepsilon}$.

Taking into account that $D_{y} \widehat{\boldsymbol{v}}=0$ a.e. in $\left\{y_{3}>M\right\}$ and that $\zeta=1$ a.e. on $\left\{x_{3}<\frac{1}{3}\right\}$, we have

$$
D \boldsymbol{v}_{\varepsilon}(x)=D \boldsymbol{v}(x)+\frac{1}{\sqrt{\varepsilon}} D_{y} \widehat{\boldsymbol{v}}\left(x^{\prime}, \frac{x}{\varepsilon}\right)+\boldsymbol{h}_{\varepsilon}(x),
$$

where, using that $\boldsymbol{v}, \widehat{\boldsymbol{v}}$ and $\zeta$ are bounded with bounded derivatives, the function $\boldsymbol{h}_{\varepsilon} \in C^{0}\left(\bar{\Omega}_{\varepsilon}\right)^{3 \times 3}$ satisfies

$$
\begin{aligned}
\left|\boldsymbol{h}_{\varepsilon}\right| \leq C \sqrt{\varepsilon}+ & C\left[\left(\frac{1}{\sqrt{\varepsilon} R_{\varepsilon}}+\frac{1}{\sqrt{\varepsilon}}\right)\left(\left|\frac{\delta_{\varepsilon}^{2}}{\varepsilon^{\frac{5}{2}}}-\mu^{2}\right|+\frac{\delta_{\varepsilon}^{2}}{\varepsilon^{2}}\right)\right. \\
& \left.+\frac{\delta_{\varepsilon}^{2}}{\varepsilon^{\frac{5}{2}}} \frac{\delta_{\varepsilon}}{\varepsilon^{\frac{3}{2}}}+\frac{\delta_{\varepsilon}^{2}}{\varepsilon^{2}}+\frac{\delta_{\varepsilon}^{2}}{\varepsilon^{2}} \frac{\delta_{\varepsilon}}{\varepsilon^{\frac{3}{2}}}+\frac{\delta_{\varepsilon}}{\varepsilon^{\frac{3}{2}}}\right] \chi_{\left\{x_{3}<\varepsilon R_{\varepsilon}\right\}} \\
+ & C\left[\frac{\delta_{\varepsilon}}{\varepsilon} \frac{1}{\varepsilon^{2} R_{\varepsilon}}+\frac{\delta_{\varepsilon}}{\varepsilon} \frac{1}{\varepsilon}+\frac{\delta_{\varepsilon}}{\varepsilon} \frac{\delta_{\varepsilon}}{\varepsilon^{2}}+\frac{\delta_{\varepsilon}}{\varepsilon}\right] \chi_{\left\{x_{3}<\varepsilon^{2} R_{\varepsilon}\right\}} \\
+ & C\left[\frac{\delta_{\varepsilon}}{\varepsilon^{\frac{3}{2}}}+\frac{\delta_{\varepsilon}^{2}}{\varepsilon^{\frac{5}{2}}}+\frac{\delta_{\varepsilon}}{\varepsilon^{\frac{1}{2}}}+\frac{\delta_{\varepsilon}}{\varepsilon^{\frac{1}{2}}} \frac{1}{\varepsilon R_{\varepsilon}}\right] \chi_{\left\{x_{3}<\varepsilon R_{\varepsilon}\right\}},
\end{aligned}
$$

a.e. in $\Omega_{\varepsilon}$. Using that $R_{\varepsilon}$ tends to infinity and that $\delta_{\varepsilon} / \varepsilon^{\frac{5}{4}}$ is bounded, we get 


$$
\begin{aligned}
\left|\boldsymbol{h}_{\varepsilon}\right| \leq C \sqrt{\varepsilon}+ & C\left[\frac{1}{\sqrt{\varepsilon}}\left(\left|\frac{\delta_{\varepsilon}^{2}}{\varepsilon^{\frac{5}{2}}}-\mu^{2}\right|+\frac{\delta_{\varepsilon}^{2}}{\varepsilon^{2}}\right)+\frac{\delta_{\varepsilon}}{\varepsilon^{\frac{3}{2}}}+\frac{\delta_{\varepsilon}^{2}}{\varepsilon^{2}}+\frac{\delta_{\varepsilon}^{2}}{\varepsilon^{2}} \frac{\delta_{\varepsilon}}{\varepsilon^{\frac{3}{2}}}\right] \chi_{\left\{x_{3}<\varepsilon R_{\varepsilon}\right\}} \\
+ & C\left[\frac{\delta_{\varepsilon}}{\varepsilon} \frac{1}{\varepsilon}+\frac{\delta_{\varepsilon}}{\varepsilon} \frac{\delta_{\varepsilon}}{\varepsilon^{2}}+\frac{\delta_{\varepsilon}}{\varepsilon}\right] \chi_{\left\{x_{3}<\varepsilon^{2} R_{\varepsilon}\right\}} \\
& +C\left[\frac{\delta_{\varepsilon}}{\varepsilon^{\frac{3}{2}}}+\frac{\delta_{\varepsilon}^{2}}{\varepsilon^{\frac{5}{2}}}+\frac{\delta_{\varepsilon}}{\varepsilon^{\frac{1}{2}}}\right] \chi_{\left\{x_{3}<\varepsilon R_{\varepsilon}\right\}} .
\end{aligned}
$$

Therefore, by (4.31) we have

$$
\begin{aligned}
\int_{\Omega_{\varepsilon}}\left|\boldsymbol{h}_{\varepsilon}\right|^{2} d x & \leq O_{\varepsilon}+C R_{\varepsilon}\left[\left(\frac{\delta_{\varepsilon}^{2}}{\varepsilon^{\frac{5}{2}}}-\mu^{2}\right)^{2}+\frac{\delta_{\varepsilon}^{2}}{\varepsilon^{2}}+\frac{\delta_{\varepsilon}^{4}}{\varepsilon^{3}}+\frac{\delta_{\varepsilon}^{6}}{\varepsilon^{6}}+\frac{\delta_{\varepsilon}^{4}}{\varepsilon^{4}}+\delta_{\varepsilon}^{2}\right] \\
& \leq O_{\varepsilon}+C R_{\varepsilon}\left[\left(\frac{\delta_{\varepsilon}^{2}}{\varepsilon^{\frac{5}{2}}}-\mu^{2}\right)^{2}+\frac{\delta_{\varepsilon}^{2}}{\varepsilon^{2}}\right]=O_{\varepsilon}
\end{aligned}
$$

Taking $\boldsymbol{v}_{\varepsilon}$ as a test function in (4.28) and using that $\left\|\boldsymbol{u}_{\varepsilon}\right\|_{H^{1}\left(\Omega_{\varepsilon}\right)^{3}},\left\|p_{\varepsilon}\right\|_{L^{2}\left(\Omega_{\varepsilon}\right)}$ are bounded, $\left\|\boldsymbol{v}_{\varepsilon}-\boldsymbol{v}\right\|_{C^{0}\left(\bar{\Omega}_{\varepsilon}\right)^{3}}$ tends to zero, (4.33) and (4.34), we get

$$
\begin{aligned}
\int_{\Omega_{\varepsilon}}\left(D \boldsymbol{u}_{\varepsilon}: D \boldsymbol{v}-p_{\varepsilon} \operatorname{div} \boldsymbol{v}\right) d x \\
\quad+\frac{1}{\sqrt{\varepsilon}} \int_{\Omega_{\varepsilon}}\left(D \boldsymbol{u}_{\varepsilon}: D_{y} \widehat{\boldsymbol{v}}\left(x^{\prime}, \frac{x}{\varepsilon}\right)-p_{\varepsilon} \operatorname{div}_{y} \widehat{\boldsymbol{v}}\left(x^{\prime}, \frac{x}{\varepsilon}\right)\right) d x+O_{\varepsilon}=\int_{\Omega_{\varepsilon}} \boldsymbol{f} \cdot \boldsymbol{v} d x .
\end{aligned}
$$

In last equality, we have used the estimate

$$
\int_{\Omega_{\varepsilon} \backslash \Omega}\left|D \boldsymbol{v}+\frac{1}{\sqrt{\varepsilon}} D_{y} \widehat{\boldsymbol{v}}\left(x^{\prime}, \frac{x}{\varepsilon}\right)\right|^{2} d x \leq \frac{C}{\varepsilon}\left|\Omega_{\varepsilon} \backslash \Omega\right| \leq C \frac{\delta_{\varepsilon}}{\varepsilon} \leq C \sqrt{\varepsilon} .
$$

Therefore, (4.35) can be rewritten as

$$
\begin{aligned}
\int_{\Omega}\left(D \boldsymbol{u}_{\varepsilon}: D \boldsymbol{v}-p_{\varepsilon} \operatorname{div} \boldsymbol{v}\right) d x \\
\quad+\frac{1}{\sqrt{\varepsilon}} \int_{\Omega}\left(D \boldsymbol{u}_{\varepsilon}: D_{y} \widehat{\boldsymbol{v}}\left(x^{\prime}, \frac{x}{\varepsilon}\right)-p_{\varepsilon} \operatorname{div}_{y} \widehat{\boldsymbol{v}}\left(x^{\prime}, \frac{x}{\varepsilon}\right)\right) d x+O_{\varepsilon}=0 .
\end{aligned}
$$

Taking into account (3.6), we deduce

$$
\begin{aligned}
\int_{\Omega}(D \boldsymbol{u}: D \boldsymbol{v}-p \operatorname{div} \boldsymbol{v}) d x \\
\quad+\frac{1}{\sqrt{\varepsilon}} \int_{\Omega}\left(D \boldsymbol{u}_{\varepsilon}: D_{y} \widehat{\boldsymbol{v}}\left(x^{\prime}, \frac{x}{\varepsilon}\right)-p_{\varepsilon} \operatorname{div}_{y} \widehat{\boldsymbol{v}}\left(x^{\prime}, \frac{x}{\varepsilon}\right)\right) d x+O_{\varepsilon}=\int_{\Omega} \boldsymbol{f} \cdot \boldsymbol{v} d x,
\end{aligned}
$$

with $\boldsymbol{v}$ and $\widehat{\boldsymbol{v}}$ satisfying (4.29). In order to estimate the second term in (4.36), we introduce the sequences $\widehat{\boldsymbol{u}}_{\varepsilon}, \widehat{p}_{\varepsilon}$ respectively defined by (4.1) and (4.2). By (3.5) 
and Lemmas 4.2 and 4.4 , we can assume that there exist $\widehat{p} \in L^{2}\left(\mathcal{T}^{2} \times \widehat{Q}\right)$ and $\widehat{\boldsymbol{u}} \in L^{2}\left(\mathcal{T}^{2} ; \mathcal{V}^{3}\right)$ which satisfy (4.5), (4.8), (4.9) and (4.11). This yields:

$$
\begin{aligned}
\frac{1}{\sqrt{\varepsilon}} & \int_{\Omega}\left(D \boldsymbol{u}_{\varepsilon}: D_{y} \widehat{\boldsymbol{v}}\left(x^{\prime}, \frac{x}{\varepsilon}\right)-p_{\varepsilon} \operatorname{div}_{y} \widehat{\boldsymbol{v}}\left(x^{\prime}, \frac{x}{\varepsilon}\right)\right) d x \\
& =\sum_{k^{\prime} \in I_{\varepsilon}} \int_{Q_{\varepsilon}^{k^{\prime}}}\left(\frac{1}{\sqrt{\varepsilon}} D \boldsymbol{u}_{\varepsilon}(x): D_{y} \widehat{\boldsymbol{v}}\left(x^{\prime}, \frac{x}{\varepsilon}\right)-\frac{1}{\sqrt{\varepsilon}} p_{\varepsilon}(x) \operatorname{div}_{y} \widehat{\boldsymbol{v}}\left(x^{\prime}, \frac{x}{\varepsilon}\right)\right) d x \\
& =\sum_{k^{\prime} \in I_{\varepsilon}} \varepsilon^{3} \int_{Q_{\varepsilon}^{k^{\prime}}}\left(\frac{1}{\sqrt{\varepsilon}} D \boldsymbol{u}_{\varepsilon}\left(\varepsilon\left(k^{\prime}+y^{\prime}\right), \varepsilon y_{3}\right): D_{y} \widehat{\boldsymbol{v}}\left(x^{\prime}, y\right)-\frac{1}{\sqrt{\varepsilon}} p_{\varepsilon}\left(\varepsilon\left(k^{\prime}+y^{\prime}\right), \varepsilon y_{3}\right) \operatorname{div}_{y} \widehat{\boldsymbol{v}}\left(x^{\prime}, y\right)\right) d y \\
& =\sum_{k^{\prime} \in I_{\varepsilon}} \varepsilon^{2} \int_{Q_{\varepsilon}^{k^{\prime}}}\left(\frac{\varepsilon}{\sqrt{\varepsilon}} D \boldsymbol{u}_{\varepsilon}\left(\varepsilon\left(k^{\prime}+y^{\prime}\right), \varepsilon y_{3}\right): D_{y} \widehat{\boldsymbol{v}}\left(x^{\prime}, y\right)-\sqrt{\varepsilon} p_{\varepsilon}\left(\varepsilon\left(k^{\prime}+y^{\prime}\right), \varepsilon y_{3}\right) \operatorname{div}_{y} \widehat{\boldsymbol{v}}\left(x^{\prime}, y\right)\right) d y \\
& =\sum_{k^{\prime} \in I_{\varepsilon}} \varepsilon^{2} \int_{Q_{\varepsilon}^{k^{\prime}}}\left(\frac{1}{\sqrt{\varepsilon}} D_{y} \widehat{\boldsymbol{u}}_{\varepsilon}\left(x^{\prime}, y\right): D_{y} \widehat{\boldsymbol{v}}\left(x^{\prime}, y\right)-\sqrt{\varepsilon} \widehat{p}_{\varepsilon}\left(x^{\prime}, y\right) \operatorname{div}_{y} \widehat{\boldsymbol{v}}\left(x^{\prime}, y\right)\right) d y \\
& =\int_{\mathcal{T}^{2}} \int_{\widehat{Q}_{M}}\left(\frac{1}{\sqrt{\varepsilon}} D_{y} \widehat{\boldsymbol{u}}_{\varepsilon}\left(x^{\prime}, y\right): D_{y} \widehat{\boldsymbol{v}}\left(x^{\prime}, y\right)-\sqrt{\varepsilon} \widehat{p}_{\varepsilon}\left(x^{\prime}, y\right) \operatorname{div}_{y} \widehat{\boldsymbol{v}}\left(x^{\prime}, y\right)\right) d x^{\prime} d y \\
= & \int_{\mathcal{T}^{2} \times \widehat{Q}_{M}}\left(D_{y} \widehat{\boldsymbol{u}}: D_{y} \widehat{\boldsymbol{v}}-\widehat{p} \operatorname{div}_{y} \widehat{\boldsymbol{v}}\right) d x^{\prime} d y+O_{\varepsilon} .
\end{aligned}
$$

Substituting in (4.36) we set

$$
\int_{\Omega}(D \boldsymbol{u}: D \boldsymbol{v}-p \operatorname{div} \boldsymbol{v}) d x+\int_{\mathcal{T}^{2} \times \widehat{Q}_{M}}\left(D_{y} \widehat{\boldsymbol{u}}: D_{y} \widehat{\boldsymbol{v}}-\widehat{p} \operatorname{div}_{y} \widehat{\boldsymbol{v}}\right) d x^{\prime} d y=\int_{\Omega} \boldsymbol{f} \cdot \boldsymbol{v} d x,
$$

for every $\boldsymbol{v} \in C_{c}^{1}\left(\mathcal{T}^{2} \times(-1,1)\right)^{3}, \widehat{\boldsymbol{v}} \in C_{c}^{1}\left(\mathcal{T}^{2} ; C^{1}(\widehat{Q})^{3}\right)$, with $D_{y} \widehat{\boldsymbol{v}}\left(x^{\prime}, y\right)=0$ a.e. in $\left\{y_{3}>M\right\}$, for some $M>0$, and such that (4.29) is satisfied. By density, this equality holds true for every $\boldsymbol{v} \in H^{1}\left(\Omega_{\varepsilon}\right)^{3}$, and every $\widehat{\boldsymbol{v}} \in L^{2}\left(\mathcal{T}^{2} ; \mathcal{V}^{3}\right)$ such that

$$
\begin{gathered}
\boldsymbol{v}=0 \quad \text { on } \Gamma_{\text {top }}, \\
v_{3}\left(x^{\prime}, 0\right)=0, \quad \widehat{v}_{3}\left(x^{\prime}, y^{\prime}, 0\right)=-\mu^{2} \partial_{y_{1}} \Psi_{1}\left(y_{1}\right) \partial_{y_{2}} \Psi_{2}\left(y_{2}\right) v_{2}\left(x^{\prime}, 0\right), \\
v_{1}\left(x^{\prime}, 0\right) \partial_{y_{1}} \Psi_{1}\left(y_{1}\right)=0, \quad \text { a.e. }\left(x^{\prime}, y^{\prime}\right) \in \mathcal{T}^{2} \times \mathcal{T}^{2} .
\end{gathered}
$$

Let us now obtain an equation for $\boldsymbol{u}$ eliminating $\widehat{\boldsymbol{u}}$ and $\widehat{p}$ in (4.37). For this purpose, we take $\boldsymbol{v}=\mathbf{0}$ in (4.37). This proves that $(\widehat{\boldsymbol{u}}, \widehat{p})$ is a solution of

$$
\left\{\begin{aligned}
-\Delta_{y} \widehat{\boldsymbol{u}}+\nabla_{y} \widehat{p}=\mathbf{0} & \text { in } \mathbb{R}^{2} \times \mathbb{R}^{+}, \\
\operatorname{div}_{y} \widehat{\boldsymbol{u}}=0 & \text { in } \mathbb{R}^{2} \times \mathbb{R}^{+}, \\
(\widehat{\boldsymbol{u}}, \widehat{p}) \in \mathcal{V}^{3} \times L^{2}(\widehat{Q}), & \\
\widehat{u}_{3}\left(x^{\prime}, y^{\prime}, 0\right)=-\mu^{2} \partial_{y_{1}} \Psi_{1}\left(y_{1}\right) \partial_{y_{2}} \Psi_{2}\left(y_{2}\right) u_{2}\left(x^{\prime}, 0\right) & \text { on } \mathbb{R}^{2} \times\{0\}, \\
-\partial_{y_{3}} \widehat{u}_{1}=0,-\partial_{y_{3}} \widehat{u}_{2}=0 & \text { on } \mathbb{R}^{2} \times\{0\},
\end{aligned}\right.
$$

a.e. in $\mathcal{T}^{2}$. Defining $\left(\widehat{\phi}^{\mu}, \widehat{q}^{\mu}\right)$ by (3.14), we deduce by linearity and uniqueness

$$
D_{y} \widehat{\boldsymbol{u}}\left(x^{\prime}, y\right)=-u_{2}\left(x^{\prime}, 0\right) D_{y} \widehat{\boldsymbol{\phi}}^{\mu}(y) \quad \text { a.e. in } \mathbb{R}^{2} \times(0,+\infty),
$$




$$
\widehat{p}\left(x^{\prime}, y\right)=u_{2}\left(x^{\prime}, 0\right) \widehat{q}^{\mu}(y) \quad \text { a. e. in } \mathbb{R}^{2} \times(0,+\infty) .
$$

Now, consider $\boldsymbol{v} \in H^{1}(\Omega)^{3}$, with $\boldsymbol{v}=0$ on $\Gamma_{\text {top }}, v_{3}=0$ on $\Gamma$. We take $\boldsymbol{v}$ and $\widehat{\boldsymbol{v}}\left(x^{\prime}, y\right)=-v_{2}\left(x^{\prime}, 0\right) \widehat{\phi}^{\mu}(y)$ as test functions in (4.37); taking into account (4.39), we get

$$
\int_{\Omega}(D \boldsymbol{u}: D \boldsymbol{v}-p \operatorname{div} \boldsymbol{v}) d x+\int_{\Gamma} M_{\mu} u_{2} v_{2} d x^{\prime}=\int_{\Omega} \boldsymbol{f} \cdot \boldsymbol{v} d x .
$$

By the arbitrariness of $\boldsymbol{v}$, this proves that $(\boldsymbol{u}, p)$ is a solution of problem (3.7)-(3.8)(3.16).

Step 2 The case $\delta_{\varepsilon} \gg \varepsilon^{\frac{5}{4}}$.

As in Step 1, we consider $\boldsymbol{v} \in C_{c}^{1}\left(\mathcal{T}^{2} \times(-1,1)\right)^{3}$, with $\boldsymbol{v}\left(x^{\prime}, x_{3}\right)=\boldsymbol{v}\left(x^{\prime}, 0\right)$ if $x_{3} \leq 0$, $v_{3}=0$ on $\Gamma$. Then, for $\zeta \in C^{\infty}(\mathbb{R})$, which satisfies (4.30), and $R_{\varepsilon}$ satisfying (4.31), we define $\boldsymbol{v}_{\varepsilon} \in H^{1}\left(\mathcal{T}^{2} \times(-1,1)\right)^{3}$ by

$$
\left\{\begin{array}{l}
v_{\varepsilon, 1}(x)=v_{1}(x) \\
v_{\varepsilon, 2}(x)=v_{2}(x) \\
v_{\varepsilon, 3}=v_{3}(x)-\frac{\delta_{\varepsilon}}{\varepsilon} \zeta\left(\frac{x_{3}}{\varepsilon^{2} R_{\varepsilon}}\right) \partial_{1} \Psi_{1}\left(\frac{x_{1}}{\varepsilon}+\frac{\delta_{\varepsilon}}{\varepsilon} \Psi_{2}\left(\frac{x_{2}}{\varepsilon}\right)\right) v_{1}(x) \\
\quad-\frac{\delta_{\varepsilon}^{2}}{\varepsilon^{2}} \zeta\left(\frac{x_{3}}{\varepsilon^{2} R_{\varepsilon}}\right) \partial_{1} \Psi_{1}\left(\frac{x_{1}}{\varepsilon}+\frac{\delta_{\varepsilon}}{\varepsilon} \Psi_{2}\left(\frac{x_{2}}{\varepsilon}\right)\right) \partial_{2} \Psi_{2}\left(\frac{x_{2}}{\varepsilon}\right) v_{2}(x) .
\end{array}\right.
$$

The sequence $\boldsymbol{v}_{\varepsilon}$ satisfies $\boldsymbol{v}_{\varepsilon} \cdot \boldsymbol{\nu}=0$ on $\Gamma$ and $\boldsymbol{v}_{\varepsilon}=0$ on $\Gamma_{\text {top }}$. We can take $\boldsymbol{v}_{\varepsilon}$ as test function in (4.28). Proceeding as in Step 1, we can prove that

$$
D \boldsymbol{v}_{\varepsilon}=D \boldsymbol{v}+\boldsymbol{h}_{\varepsilon}
$$

with $\boldsymbol{h}_{\varepsilon}$ satisfying

$$
\int_{\Omega_{\varepsilon}}\left|\boldsymbol{h}_{\varepsilon}\right|^{2} d x \leq O_{\varepsilon} .
$$

Then, passing to the limit in $\varepsilon$ and reasoning by density we get

$$
\left\{\begin{array}{c}
\int_{\Omega} D \boldsymbol{u}: D \boldsymbol{v} d x-\int_{\Omega} p \operatorname{div} \boldsymbol{v} d x=0 \quad \forall \boldsymbol{v} \in H^{1}(\Omega)^{3} \\
v_{3}=0 \text { on } \Gamma, \quad \boldsymbol{v}=\mathbf{0} \text { on } \Gamma_{\text {top }}, \\
v_{1}\left(x^{\prime}, 0\right) \partial_{1} \Psi_{1}\left(y_{1}\right)=0, \quad v_{2}\left(x^{\prime}, 0\right) \partial_{1} \Psi_{1}\left(y_{1}\right) \partial_{2} \Psi_{2}\left(y_{2}\right)=0 \text { a.e. }\left(x^{\prime}, y^{\prime}\right) \in \mathcal{T}^{2} \times \mathcal{T}^{2} .
\end{array}\right.
$$

This is equivalent to problem (3.7)-(3.8)-(3.17).

Proof of Proposition 3.4. It results from Lemma 4 in [2], applied to $\left(\widehat{\boldsymbol{\phi}}^{\lambda}-\boldsymbol{V}^{\lambda}, \widehat{p}^{\lambda}\right)$, where $\boldsymbol{V}^{\lambda}$ is a constant vector defined by

$$
V_{i}^{\lambda}=\int_{\mathcal{T}^{2}} \widehat{\phi}_{i}^{\lambda}\left(y^{\prime}, 0\right) d y^{\prime}, i=1,2, \quad V_{3}^{\lambda}=0 .
$$


This translation ensures that the vector field $\widehat{\phi}^{\lambda}-\boldsymbol{V}^{\lambda}$ satisfies the following property:

$$
\int_{\mathcal{T}^{2}}\left(\widehat{\phi}^{\lambda}\left(y^{\prime}, 0\right)-\boldsymbol{V}^{\lambda}\right) d y^{\prime}=0
$$

Notice that, by periodicity, the boundary condition satisfied by $\widehat{\phi}_{3}^{\lambda}$ on $y_{3}=0$ yields $\int_{\mathcal{T}^{2}} \widehat{\phi}_{3}^{\lambda}\left(y^{\prime}, 0\right) d y^{\prime}=0$.

\section{Numerical results}

In this section, we simulate numerically the asymptotic effect of wavy riblets on a viscous fluid, in the streamwise direction. To this aim, we consider a flow driven by a constant external force $\boldsymbol{f}=(0,1,0)$ and modeled by Stokes equations (3.1) associated with boundary conditions (3.2), (3.3), (3.4), and periodic in the lateral coordinates. In order to capture the influence of fast oscillations of the ribbed boundary, we simulate the homogenized system described by Theorem 3.1. Since we focus on the streamwise component of the fluid velocity, we consider the second critical case, namely, $\delta_{\varepsilon} \sim \mu \varepsilon^{5 / 4}$ with $0<\mu<+\infty$. In that case, the homogenized velocity field $\boldsymbol{u}$ is the solution to Stokes equations (3.7) in the unit cube $\Omega$, completed with boundary conditions (3.8), (3.16). Boundary condition (3.16), satisfied by the streamwise component of the velocity, introduces an extra friction term $M_{\mu}$ defined by (3.15). As a result, the numerical approximation of $\boldsymbol{u}$ is determined by the resolution of the auxiliary problem (3.14), that allows for the computation of this friction coefficient.

This section is organized as follows. In Subsection 5.1, we present and justify the numerical approximation of the solution to the auxiliary problem (3.14). In Subsection 5.2 , we define the geometry of the wavy riblets that we consider in the simulations. Finally, Subsection 5.3 is devoted to the statement and discussion of the results.

\subsection{Numerical approximation of auxiliary system (3.14)}

In order to simulate system (3.14), we fix a truncature parameter $H>0$ and we approximate the functions $\left(\widehat{\phi}^{\mu}, \widehat{q}^{\mu}\right)$ on $\mathcal{T}^{2} \times(0, H)$, as follows. We define $\left(\widehat{\phi}_{H}^{\mu}, \widehat{q}_{H}^{\mu}\right) \in$ $H^{1}\left(\mathbb{R}^{2} \times(0, H), \mathbb{R}^{3}\right) \times L^{2}\left(\mathbb{R}^{2} \times(0, H)\right)$, as the solution of

$$
\left\{\begin{array}{rc}
-\Delta_{y} \widehat{\phi}_{H}^{\mu}+\nabla_{y} \widehat{q}_{H}^{\mu}=\mathbf{0} & \text { in } \mathbb{R}^{2} \times(0, H), \\
\operatorname{div}_{y} \widehat{\boldsymbol{\phi}}_{H}^{\mu}=0 & \text { in } \mathbb{R}^{2} \times(0, H), \\
\widehat{\phi}_{H, 3}^{\mu}\left(y^{\prime}, 0\right)=\mu^{2} \partial_{1} \Psi_{1}\left(y_{1}\right) \partial_{2} \Psi_{2}\left(y_{2}\right) & \text { on } \mathbb{R}^{2} \times\{0\}, \\
-\partial_{3} \widehat{\phi}_{H, 1}^{\mu}=0,-\partial_{3} \widehat{\phi}_{H, 2}^{\mu}=0 & \text { on } \mathbb{R}^{2} \times\{0\}, \\
\partial_{3} \widehat{\phi}_{H, 1}^{\mu}\left(y^{\prime}, H\right)=0, & \\
\partial_{3} \widehat{\phi}_{H, 2}^{\mu}\left(y^{\prime}, H\right)=0, & \\
\partial_{3} \widehat{\phi}_{H, 3}^{\mu}-\widehat{q}_{H}^{\mu}=0 & \text { on } \mathbb{R}^{2} \times\{H\},
\end{array}\right.
$$

and the corresponding approximate value of the coefficient $M_{\mu, H}$ by

$$
M_{\mu, H}=\int_{\mathcal{T}^{2} \times(0, H)}\left|D_{y} \widehat{\phi}_{H}^{\mu}\right|^{2} d y
$$


Justification of the approximation. The validity of the approximate system (5.1) is strongly related to the exponential decay properties (3.22). Assume, for instance, that $H>1$. We denote by $\boldsymbol{F}_{H} \in C^{\infty}\left(\mathcal{T}^{2}\right)^{3}$ the function defined by

$$
\boldsymbol{F}_{H}\left(y^{\prime}\right):=\partial_{3} \widehat{\phi}^{\lambda}\left(y^{\prime}, H\right)-\widehat{q}^{\lambda}\left(y^{\prime}, H\right) e_{3} \quad \forall\left(y_{1}, y_{2}\right) \in \mathcal{T}^{2} .
$$

Applying property (3.22) with $\alpha=1, t=1$, we obtain the existence of a constant $C>0$ such that

$$
\left\|\boldsymbol{F}_{H}\right\|_{L^{2}\left(\mathcal{T}^{2}\right)^{3}} \leq C \mu^{2}\left\|\partial_{1} \Psi_{1}\right\|_{L^{2}\left(\mathcal{T}^{1}\right)}\left\|\partial_{2} \Psi_{2}\right\|_{L^{2}\left(\mathcal{T}^{1}\right)} \mathrm{e}^{-\tau H}
$$

In order to compare the exact solution $\left(\widehat{\phi}^{\mu}, \widehat{q}^{\mu}\right)$ with the approximation $\left(\widehat{\phi}_{H}^{\mu}, \widehat{q}_{H}^{\mu}\right)$, we notice that $\left(\widehat{\phi}^{\mu}, \widehat{q}^{\mu}\right)$ is a solution to system $(5.1)$ in $\mathcal{T}^{2} \times(0, H)$, but with a slightly modified boundary condition on $y_{3}=H$, namely,

$$
\partial_{3} \widehat{\phi}^{\mu}-\widehat{q}^{\mu} e_{3}=\boldsymbol{F}_{H} \quad \text { on } \quad y_{3}=H .
$$

As a result, the difference $\left(\widehat{\phi}^{\mu}-\widehat{\phi}_{H}^{\mu}, \widehat{q}^{\mu}-\widehat{q}_{H}^{\mu}\right)$ is a solution to a Stokes system in $\mathcal{T}^{2} \times(0, H)$, with the following boundary conditions:

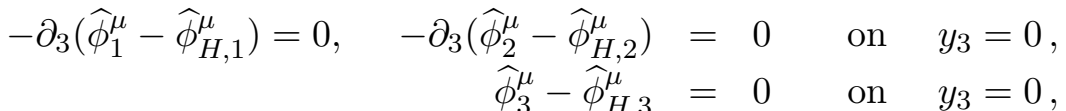

$$
\begin{aligned}
& \partial_{3}\left(\widehat{\boldsymbol{\phi}}^{\mu}-\widehat{\boldsymbol{\phi}}_{H}^{\mu}\right)-\left(\widehat{q}^{\mu}-\widehat{q}_{H}^{\mu}\right) e_{3}=\boldsymbol{F}_{H} \quad \text { on } \quad y_{3}=H .
\end{aligned}
$$

It follows that there exists a constant $C>0$ such that

$$
\left\|D\left(\widehat{\phi}^{\mu}-\widehat{\phi}_{H}^{\mu}\right)\right\|_{L^{2}\left(\mathcal{T}^{2} \times(0, H)\right)} \leq C \mu^{2}\left\|\partial_{1} \Psi_{1}\right\|_{L^{2}\left(\mathcal{T}^{1}\right)}\left\|\partial_{2} \Psi_{2}\right\|_{L^{2}\left(\mathcal{T}^{1}\right)} \mathrm{e}^{-\tau H} .
$$

Numerical method. In order to compute the solution to system (5.1), we use a finite element discretization of the problem, using FreeFem ++ software [21]. We consider the Taylor-Hood approximation for the velocity-pressure pair, i.e., $P_{2}$ elements for the velocity field and $P_{1}$ elements for the pressure. It is well known that this choice is compatible with the Babuška-Brezzi condition [20], [5]. The three-dimensional mesh of the fluid domain $\mathcal{T}^{2} \times(0, H)$ is obtained by a constrained Delaunay tetrahedralization. Finally, the incompressibility condition is approximated by the standard penalty formulation

$$
\int_{\Omega}\left(\operatorname{div} \widehat{\phi}^{\mu}+\eta \widehat{q}^{\mu}\right) \widehat{r}=0 \quad \forall \widehat{r} \in Q_{h}
$$

where $Q_{h}$ is the discrete approximation space for the pressure, and $\eta=10^{-6}$ is the penalty parameter [20], [22].

\subsection{Definition of the geometry of the wavy riblets}

The geometry of the wavy riblets is determined by the choice of the profile functions $\Psi_{1}, \Psi_{2}$. To perform comparisons, and in view of estimate (5.3), we normalize functions $\Psi_{1}, \Psi_{2}$ by setting

$$
\left\|\partial_{1} \Psi_{1}\right\|_{L^{2}\left(\mathcal{T}^{1}\right)}=1, \quad\left\|\partial_{2} \Psi_{2}\right\|_{L^{2}\left(\mathcal{T}^{1}\right)}=1 .
$$

Choice of $\Psi_{2}$. We consider a sinusoidal function

$$
\Psi_{2}\left(y_{2}\right)=K_{2} \sin \left(2 \pi y_{2}\right),
$$

where $K_{2}$ is the normalization constant. 
Choice of $\Psi_{1}$. The functions $\Psi_{1}$ that we consider are obtained by regularizing riblet profiles that are widely used in the experimental studies [18]:

(i) the $V$-shape riblets, or sawtooth riblets;

(ii) the $U$-shape riblets, or scallop riblets;

(iii) the blade riblets.

For each of these profiles, we have applied a parabolic regularization in the vicinity of each singular point, obtaining functions $\Psi_{1}$ of class $C^{2}$ (see Figure 4 ). Finally, Figure 5 provides an outline of the associated profiles $\Psi_{\varepsilon}$, restricted to the line $\mathbb{R} \times\{0\}$.
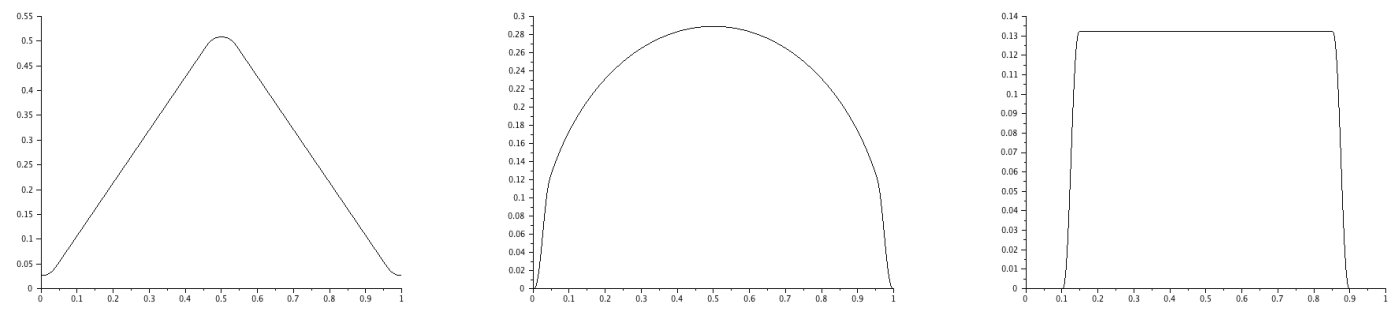

Figure 4: Functions $\Psi_{1}$ associated with $V$-shaped, $U$-shaped and blade riblets (from left to right)
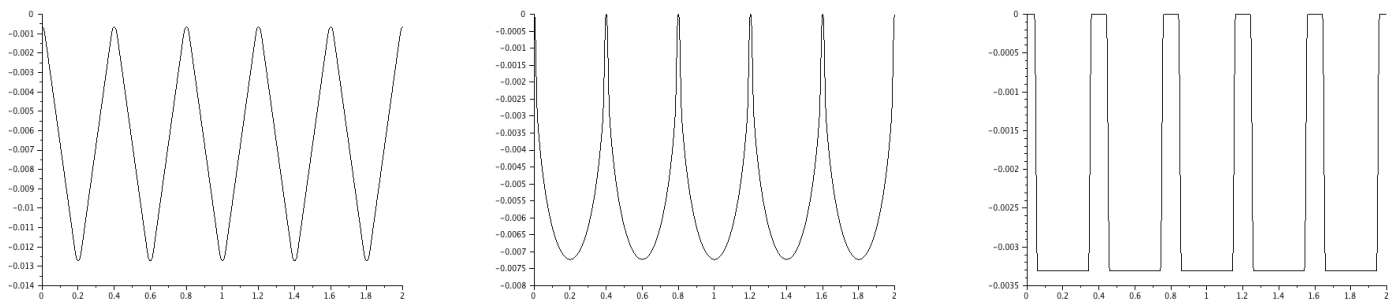

Figure 5: Functions $\Psi_{\varepsilon}$ associated with $V$-shaped, $U$-shaped and blade riblets (from left to right)

\subsection{Results and discussion}

For each type of geometry of riblets described in Subsection 5.2, we compute an approximate value of the friction coefficient $M_{\mu}$, for different values of $\mu$. To this aim, we start by solving system (5.1) in a domain of height $H=3$, for a fixed value $\mu=1$. The corresponding results are given in Table 1.

Then, for any value of $\mu$, we set $M_{\mu, H}=\mu^{4} M_{1, H}$, which is the numercial counterpart of the relation $M_{\mu}=\mu^{4} M_{1}$, satisfied by the exact friction coefficient as a direct consequence of (3.15).

Then, for each value of $M_{\mu, H}$, we solve by a finite element method (see Subsection 5.1 for details) the homogenized Stokes system 


\begin{tabular}{|c|c|c|c|}
\hline & $V$-shape & $U$-shape & Blade \\
\hline$M_{\mu, H}$ & 311.516 & 1270.89 & 1865.94 \\
\hline
\end{tabular}

Table 1: Approximate values of the friction coefficient $M_{\mu, H}$, with $\mu=1$, and $H=3$, for the $U$-shape, $V$-shape and blade riblets.

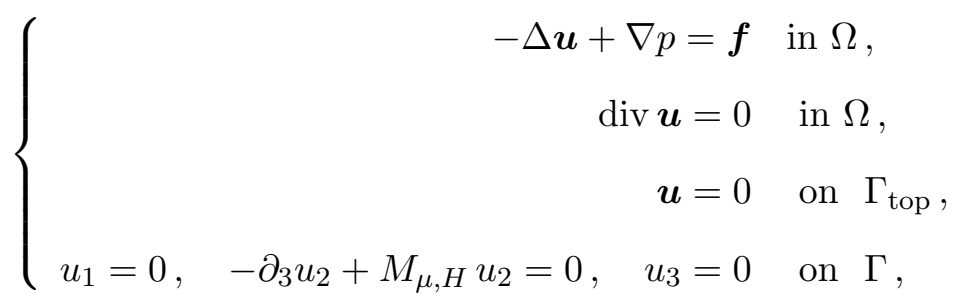

where $\Omega=\mathcal{T}^{2} \times(0,1), \Gamma=\mathcal{T}^{2} \times\{0\}, \Gamma_{\text {top }}=\mathcal{T}^{2} \times\{1\}$, in the particular case $\boldsymbol{f}=(0,1,0)$. We define the average streamwise velocity $\overline{u_{2}}$ by

$$
\overline{u_{2}}=\int_{\Omega} u_{2}=\int_{\Omega} \boldsymbol{f} \cdot \boldsymbol{u} .
$$

The results are plotted in Figure 6.

We observe that for each riblet profile, there exists a transition between the perfect slip regime, associated with the limit $\mu \rightarrow 0$, and the no-slip regime $\mu \rightarrow+\infty$. During this transition, the values of the average streamwise velocities that we observe for the $V$-shape, the $U$-shape and the blade riblets, are ordered in a way that is consistent with the values of the approximate friction coefficients given in Table 1. The $V$-shape riblet, associated with the smallest friction coefficient, corresponds to the highest velocity of the flow; on the opposite, the $U$-shape and the blade riblets, whose friction coefficients at $\mu=1$ are 4 to 6 times larger, have a stronger slowing effect on the flow.

This significant difference between the $V$-shape riblet on the one hand, and the $U$ shape and blade riblets on the other hand, can be put in perspective with the well-known experimental results on straight riblets, that demonstrate the efficiency of thin protrusions in the reduction of the drag for turbulent flows (see [25] and the references therein). In our study, such fast and well-localized variations of the boundary give rise to a high $L^{1}$ norm of the derivative $\partial_{1} \Psi_{1}$; combined with sinusoidal oscillations of the boundary along the streamwise direction, they generate high friction coefficients $M_{\mu}$. Such high values of the friction coefficients could play a decisive role in the stabilization of the flow in the vicinity of the wall, in the case of flows at larger Reynolds numbers. This question will be addressed in a future work, by applying our homogenization results to certain turbulent models used in Large Eddy Simulation.

\section{Acknowledgements.}

The research of Francisco J. Suárez-Grau has been partially supported by the project MTM2014-53309-P of the "Ministerio de Economía y Competitividad", by the project FQM309 of the "Junta de Andalucía", and by the City of Paris postdoc research grants of the program "Research in Paris 2014". The research of Giordano Tierra has been partially supported by ERC-CZ project LL1202 (Ministry of Education, Youth and Sports of the Czech Republic). 


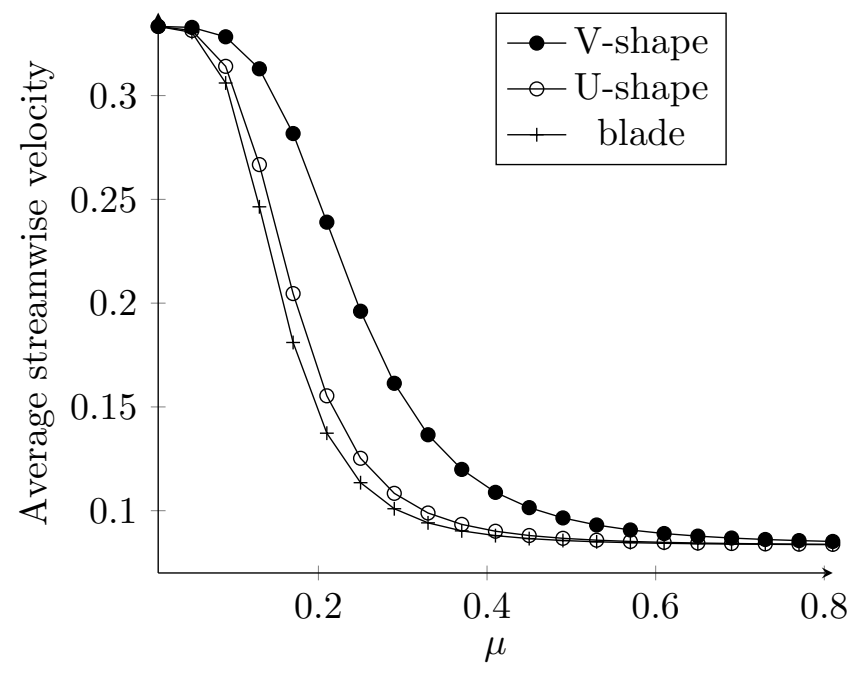

Figure 6: Average streamwise velocity $\overline{u_{2}}$ plotted against $\mu$, for a flow governed by Stokes equations (5.4).

\section{References}

[1] G. Allaire, Homogeneization and two-scale convergence, SIAM J. Math. Anal., 23 (1992), pp. 1482-1518.

[2] Y. Amirat, J. Simon, Influence de la rugosit en hydrodynamique laminaire, C. R. Acad. Sci. Paris, sr. I, 323 (1996), 313-318.

[3] T. Arbogast, J. Douglas, U. Hornung, Derivation of the double porosity model of single phase flow via homogenization theory, SIAM J. Math. Anal., 21 (1990), pp. 823-836.

[4] M. Bonnivard, D. Bucur, The uniform rugosity effect. J. Math. Fluid Mech. 14 (2012), pp 201-215.

[5] F. Brezzi, M. Fortin, Mixed and Hybrid Finite Element Methods. Springer Verlag, 1991.

[6] J. Březina, Asymptotic properties of solutions to the equations of incompressible fluid mechanics, Journal of Mathematical Fluid Mechanics, 124 (2010), pp. 536-553.

[7] D. Bucur, E. Feireisl, N. Nečasová, On the Asymptotic Limit of Flows Past a Ribbed Boundary, Journal of Mathematical Fluid Mechanics, 10 4, (2008) 554 - 568.

[8] D. Bucur, E. Feireisl, N. Nečasová, Influence of wall roughness on the slip behaviour of viscous fluids, Proc. Royal Soc. Edinburgh 138 A (2008), pp 957 - 973.

[9] D. Bucur, E. Feireisl, N. Nečasová, Boundary behaviour of viscous fluids: Influence of wall roughness and friction-driven boundary conditions, Archiv. Rational Mech. Anal., 197 (2010), pp. 117-138. 
[10] D. Bucur, E. Feireisl, S. Nečasová, J. Wolf, On the asymptotic limit of the Navier-Stokes system on domains with rough boundaries, J. Differential Equations, 244 (2008), pp. 2890-2908.

[11] J. Casado-Díaz, Two-scale convergence for nonlinear Dirichlet problems in perforated domains, Proc. Roy. Soc. Edimburgh, 130 A (2000), pp. 249-276.

[12] J. Casado-Díaz, Exponential decay for the solutions of nonlinear elliptic systems posed in unbounded cylinders, J. Math. Anal. Appl., 328 (2007), pp. 151-169.

[13] J. Casado-Díaz, E. Fernández-Cara, J. Simon, Why viscous fluids adhere to rugose walls: A mathematical explanation, J. Differential Equations, 189 (2003), pp. 526-537.

[14] J. Casado-Díaz, M. Luna-Laynez, J.D. Martín-Gómez, An adaptation of the multiscale methods for the analysis of very thin reticulated structures, C. R. Acad. Sci. Paris, Sér. I, 332 (2001), pp. 223-228.

[15] J. Casado-Díaz, M. Luna-Laynez, F.J. Suárez-Grau, Asymptotic behaviour of a viscous fluid with slip boundary conditions on a slightly rough wall, Math. Mod. Meth. Appl. Sci. 20, (2010), pp. 121-156.

[16] D. Cioranescu, A. Damlamian, G. Griso, Periodic unfolding and homogenization, C.R. Acad. Sci. Paris, Ser. I, 335 (2002), 99-104.

[17] A.L. Dalibard, D. Gérard-Varet, Effective boundary condition at a rough surface starting from a slip condition. Journal of Differential Equations 251 (2011) 3450-3487.

[18] B. Dean, B. Bhushan, Shark-skin surfaces for fluid-drag reduction in turbulent flow: a review. Phil. Trans. R. Soc. A (2010) 368, 4775-4806.

[19] D. Gérard-Varet, N. Masmoudi, Relevance of the slip condition for fluid flows near an irregular boundary. Communications in Mathematical Physics 295 (2010) 99-137.

[20] V. Girault, P.-A. Raviart, Finite element methods for Navier-Stokes equations, Springer Series in Computational Mathematics, vol. 5, Springer-Verlag, Berlin, 1986, Theory and Algorithms. MR 851383 (88b:65129).

[21] F. Hecht, New development in FreeFem++. J. Numer. Math. 20 (2012) 251-265.

[22] K. Ito, K. Kunisch, Lagrange multiplier approach to variational problems and applications, Advances in Design and Control, vol. 15, Society for Industrial and Applied Mathematics (SIAM), Philadelphia, PA, 2008. MR 2441683 (2009g:49001).

[23] W. Jaeger, A. Mikelić. On the roughness-induced effective boundary conditions for an incompressible viscous flow. J. Differential Equations, 170 (2001) 96-122.

[24] W. Jaeger, A. Mikelić. Couette Flows over a Rough Boundary and Drag Reduction. Comm. Math. Phys., 232 (2003), 429-455.

[25] F. Kramer, R. Grueneberger, E. Wassen, W. Hage, R. Meyer, Wavy riblets for turbulent drag reduction, AIAA 5th flow control conference, AIAA-2010-4583 (2010). 
[26] B. Mohammadi, O. Pironneau, F. Valentin, Rough boundaries and wall laws. Finite elements in fluids. Internat. J. Numer. Methods Fluids 27 no. 1-4 (1998), Special Issue, $169-177$.

[27] C.L.M.H. Navier, Mémoire sur les lois du mouvement des fluides. Mem. Acad. R. Sci. Paris, 6 (1823), pp 389-416.

[28] G. Nguetseng, A general convergence result for a functional related to the theory of homogenization, SIAM J. Math. Anal., 20 (1989), pp. 608-623.

[29] C. Viotti, M. Quadrio, and P. Luchini, Streamwise oscillation of spanwise velocity at the wall of a channel for turbulent drag reduction, Phys. Fluids, vol. 21, 115109 (2009).

[30] E. Wassen, R. Grueneberger, F. Kramer, W. Hage, R. Meyer, F. Thiele, Turbulent drag reduction by oscillating riblets, AIAA 4th flow control conference, AIAA-20083771 (2008). 